\title{
Soft masses in superstring models with anomalous $U(1)$ symmetries
}

\author{
Claudio A. Scrucca \\ Institut de Théorie des Phénomènes Physiques \\ Ecole Polytechnique Fédérale de Lausanne \\ CH-1015 Lausanne, Switzerland \\ E-mail: claudio.scrucca@epfl.ch
}

\begin{abstract}
We analyze the general structure of soft scalar masses emerging in superstring models involving anomalous $U(1)$ symmetries, with the aim of characterizing more systematically the circumstances under which they can happen to be flavor universal. We consider both heterotic orbifold and intersecting brane models, possibly with several anomalous and non-anomalous spontaneously broken $U(1)$ symmetries. The hidden sector is assumed to consist of the universal dilaton, Kähler class and complex structure moduli, which are supposed to break supersymmetry, and a minimal set of Higgs fields which compensate the Fayet-Iliopoulos terms. We leave the superpotential that is supposed to stabilize the hidden sector fields unspecified, but we carefully take into account the relations implied by gauge invariance and the constraints required for the existence of a metastable vacuum with vanishing cosmological constant. The results are parametrized in terms of a constrained Goldstino direction, suitably defined effective modular weights, and the $U(1)$ charges and shifts. We show that the effect induced by vector multiplets strongly depends on the functional form of the Kähler potential for the Higgs fields. We find in particular that whenever these are charged matter fields, like in heterotic models, the effect is non-trivial, whereas when they are shifting moduli fields, like in certain intersecting brane models, the effect may vanish.
\end{abstract}




\section{Introduction}

Superstring models represent a very appealing possibility for the microscopic framework underlying supersymmetric extensions of the standard model. In this respect, a crucial question concerns the way in which spontaneous supersymmetry breaking is realized. The standard paradigm is that this breaking occurs in a hidden sector and is then transmitted only through suppressed gravitational interactions to the visible sector containing the supersymmetric extension of the standard model $[1,2,3]$ (see also $[4,5,6]$ ). Since the scale of supersymmetry breaking must be much lower than the Planck scale, it is possible to study the problem within a low-energy effective supergravity description. The structure of the soft supersymmetry breaking terms depends however on the structure of certain higher-dimensional operators, as well as on the direction of supersymmetry breaking. A particularly important issue is the flavor structure of the soft scalar masses. For phenomenological reasons, these should be nearly universal, or suitably aligned with the ordinary fermion masses. It is then natural to explore how this could come about.

A natural candidate for the hidden sector of string models is that of the universal neutral moduli fields, whose scalar Vacuum Expectation Values (VEV) control the strength of the coupling and the geometry of the internal space [7, 8, 9]. An interesting point about this assumption is that the Kähler potentials for these moduli fields are determined by dimensional reduction and are universal, at leading order in the weak-coupling and low-energy expansions. They turn out to define constant curvature coset Kähler manifolds of the type $G / H$, where $G$ is a group of global isometries and $H$ is a local stability subgroup. Moreover, also the couplings between matter fields and moduli fields, which control soft scalar masses, have in general a special structure. More precisely, the leading higher-dimensional operators that are relevant for these soft masses can be parametrized in terms of certain effective modular weights for matter fields, which are constant in the simplest cases but can in general depend on the moduli fields. The superpotential that could be at the origin of the spontaneous breaking of supersymmetry is on the other hand less understood. One can however leave it unspecified, and only assume that it produces a scalar potential admitting a stationary point where the energy is approximately zero and all the moduli are stabilized with a positive squared mass. The situation can then be parametrized in terms of the Goldstino vector of auxiliary fields, which has a length that is fixed by the condition of vanishing cosmological constant and a direction that can a priori be arbitrary but will be constrained to certain cones by the metastability condition $[10,11,12]$. The structure of soft terms can then be parametrized in terms of the Goldstino direction and the effective modular weights.

An important additional ingredient, which occurs in essentially all known models and can significantly change the situation, is the presence of additional $U(1)$ gauge symmetries. For phenomenological reasons, these must be spontaneously broken, with a sufficiently large mass for the corresponding gauge bosons. A beautiful way to make this breaking natural is provided by anomalies in these extra $U(1)$ symmetries, which are also pretty 
endemic. These anomalies are cancelled through the Green-Schwarz mechanism [13], which has a slightly different form in different models, but rests on the same general structure. The basic point is that one-loop effects do not only induce anomalies, but also corrections to the Kähler potential, which force some of the moduli $M^{i}$, which were neutral at the tree level, to acquire at one-loop order a non-trivial behavior under the $U(1)$ transformations, shifting them by some constants $\delta_{i a}$. This implies then a nontrivial gauge variation of the gauge-kinetic functions depending on these moduli, which cancels the anomalies. This mechanism implies the emergence of moduli-dependent FayetIliopoulos terms, which act as a sources for the $D$-term contribution to the scalar potential once these moduli are stabilized at constant values [14]. In all known models, there exist however additional chiral multiplets with appropriate $U(1)$ charges that get VEV to approximately compensate such sources in the $D$-term potential, in such a way that the main effect of the Fayet-Iliopoulos terms is to break the $U(1)$ symmetries and to preserve supersymmetry, rather than vice-versa. In such a situation, the anomalous $U(1)$ symmetries are necessarily non-linearly realized, and the corresponding gauge bosons are massive. The Higgs fields are mostly a mixture of the charged fields taking VEV, with a small contamination from the moduli that can be neglected at leading order in $\delta_{i a}$, and the squared mass of the $U(1)$ vector bosons is of the order of a loop factor times the Planck mass squared. Furthermore, also non-anomalous $U(1)$ factors can be forced to be broken, if the mentioned Higgs fields have non-vanishing charges with respect to them. In fact, in minimal situations where there are as many Higgs fields as $U(1)$ symmetries, the VEV of the Higgs fields are entirely determined by the various $D$-flatness conditions, which do or do not have moduli-dependent Fayet-Iliopoulos source terms depending on whether the corresponding symmetry is anomalous or not.

It has been appreciated for already some time that the presence of vector multiplets with masses below the Planck scale induces important additional effects mixing the visible and the hidden sectors, if both the visible and the hidden sector fields have non-vanishing charges $[15,16]$. In fact, they give a sizable additional contribution to the soft scalar masses $m^{2}$, in spite of the fact that the auxiliary fields of these vector multiplets are suppressed as $D \sim m_{3 / 2}^{2} M_{\mathrm{P}}^{2} / M_{V}^{2}$, and thus much smaller than the auxiliary fields of the hidden sector chiral multiplets, which are of order $F \sim m_{3 / 2} M_{\mathrm{P}}$. The reason is that if both the visible and the hidden sector fields are charged, there is a contribution to $m^{2}$ that is proportional to $D$, coming from the direct minimal renormalizable coupling to the vector multiplet, and which is of the same size as the ordinary contribution proportional to $F^{2} / M_{\mathrm{P}}^{2}$, coming from non-renormalizable effective interactions with the hidden sector chiral multiplets induced by gravity. This additional contribution can also be understood as coming from additional operators that are induced in the low-energy effective theory for the light chiral multiplets when integrating out the heavy vector multiplets, with $D \sim F^{2} / M_{V}^{2}$. In general this effect cannot be neglected, and brings a dependence on the $U(1)$ charges in the soft scalar masses. This represents an additional potential source of flavor non-universality, since the charges are not necessarily flavor universal, and the 
broken symmetries might even be related to flavor physics. It is therefore important to study the structure of soft scalar masses in the presence of such non-linearly realized $U(1)$ symmetries, to determined how much these change the situation with respect to the flavor problem.

The effects on soft terms of a heavy vector field associated to an anomalous $U(1)$ symmetry were first explored in [17, 18, 19], mostly within a simplified effective set up and assuming that supersymmetry breaking originates from gaugino condensation. The structure of soft scalar masses in string models with anomalous $U(1)$ factors, with a more general supersymmetry breaking sector and no assumption on the origin of supersymmetry breaking, has instead been first studied in [20,21] and in [22, 23, 24], with slightly different points of view. However, in these papers it was implicitly assumed that the hidden sector superpotential does not depend on the charged fields Higgsing the $U(1)$ symmetries. We believe that this is not appropriate, because the moduli fields participating in the Green-Schwarz mechanism shift under gauge transformations, and a non-trivial superpotential leading to generic $F$ auxiliary fields can be gauge-invariant only if it depends on holomorphic gauge-invariant combinations of moduli fields and charged Higgs fields. In fact, in the minimal case, the relative effect of the Higgs fields compared to the moduli fields in supersymmetry breaking is completely fixed by gauge invariance. Subsequently, a proper computation of the form of soft scalar masses in the presence of an arbitrary but gauge-invariant superpotential has been performed in [25], for the minimal situation involving a modulus field and a matter Higgs field transforming respectively with a shift and a phase under an anomalous $U(1)$ symmetry. More recently, this analysis was generalized to include also additional non-shifting moduli [26]. But a general and complete discussion of the detailed form of the scalar masses in string models with possibly several broken $U(1)$ symmetries and its implications for the flavor problem is still missing.

The aim of this paper is to examine the structure of soft scalar masses in various kinds of string models involving spontaneously broken $U(1)$ symmetries, leaving the superpotential that is supposed to stabilize the moduli unspecified, but paying attention to the constraints implied by its gauge invariance. In our analysis, we will take into account the constraints that are put on the Goldstino direction by the requirement that all the moduli fields should be stabilized with a positive squared mass $[10,11,12]$. We will also generalize previous studies to cases involving several anomalous and non-anomalous $U(1)$ symmetries, and Higgs fields that are either matter fields with a canonical quadratic Kähler potential or moduli fields with a non-canonical Kähler potential. Finally, we will examine more closely the typical situations arising in heterotic and brane models. In heterotic orbifold models, the stabilization of the dilaton modulus implies a Fayet-Iliopoulos term that is compensated by a canonical charged matter Higgs fields. This leads unavoidably to a non-trivial $D$-term contribution to soft scalar masses. In intersecting brane models, on the other hand, the stabilization of the dilaton and complex structure moduli may or may not generate Fayet-Iliopoulos terms, depending on whether the angles preserve or not some $U(1)$ R-symmetry. In the second case, the net Fayet-Iliopoulos terms induced 
by the moduli fields are again compensated by canonical charged matter Higgs fields, and the situation is very similar to that of heterotic models. In the former case, on the other hand, one can interpret the complex structure moduli as non-canonical shifting moduli Higgs fields compensating the Fayet-Iliopoulos terms induced by the dilaton, and the situation is radically different. Most importantly, there exist in this case situations where the $D$-term contribution to soft scalar masses vanishes identically, as a consequence of the related functional forms of the Kähler potentials for the various involved moduli fields.

The paper is organized as follows. In section 2, we review some general results concerning scalar masses in supergravity models. In section 3, we examine more specifically the effects of heavy vector multiplets. In sections 4 and 5 we study the $F$ - and $D$-term contributions to soft scalar masses in models with $U(1)$ symmetries broken at a high scale, for general situations where the Fayet-Iliopoulos terms are approximately cancelled by the VEV of a minimal set of Higgs fields with respectively a canonical and non-canonical Kähler potential. In section 6 we apply these general results to string models with a supersymmetry breaking sector identified with the untwisted moduli sector, focusing in particular on heterotic orbifolds and intersecting brane models. Finally, in section 7 we summarize our conclusions.

\section{Soft scalar masses in supergravity models}

We will start by briefly reviewing some of the salient features of supergravity models that will be needed for our analysis [27, 28, 29, 30,31]. Let us consider more specifically a generic supergravity theory involving some chiral multiplets $Z^{r}$ and some $U(1)$ vector multiplets $V^{a}$. Setting $M_{\mathrm{P}}=1$, the theory is specified by a real Kähler and gaugeinvariant function $G=K+\ln |W|^{2}$, depending on $Z^{r}, \bar{Z}^{r}$ and $V^{a}$, and a holomorphic gauge-invariant gauge-kinetic function $f_{a b}$, depending on $Z^{r}$. Derivatives with respect to the fields $Z^{r}, \bar{Z}^{r}$ and $V^{a}$ are denoted by lower indices $r, \bar{r}$ and $a$, which are raised through the inverse of the Kähler metric $g_{r \bar{s}}=G_{r \bar{s}}$, and the inverse of the real part of the gauge kinetic function $h_{a b}=\operatorname{Re} f_{a b}$.

The $U(1)$ gauge transformations are specified by holomorphic Killing vectors $X_{a}$, generating isometries of the scalar manifold, with components $X_{a}^{r}$ depending on the fields $Z^{r}$. More precisely, the supergauge transformations of the chiral and vector superfields are given by $\delta Z^{r}=\Lambda^{a} X_{a}^{r}$ and $\delta V^{a}=-i\left(\Lambda_{a}-\bar{\Lambda}_{a}\right)$. The function $G$ must be invariant. This implies that $G_{a}=-i X_{a}^{r} G_{r}=i X_{a}^{\bar{r}} G_{\bar{r}}$. Taking derivatives, one then also deduces that $X_{a r}=-i G_{a r}$, showing that $G_{a}$ represent Killing potentials. The function $f_{a b}$ must instead have a gauge variation that matches possible residual quantum anomalies $Q_{a b c}$ : $\delta f_{a b}=i \Lambda^{c} Q_{a b c}$. This implies that $h_{a b r}=i / 2 X_{r}^{c} Q_{a b c}$.

The potential for the complex scalar fields of the chiral multiplets, which is the crucial quantity controlling spontaneous supersymmetry breaking, has the following form:

$$
V=e^{G}\left(G^{r} G_{r}-3\right)+\frac{1}{2} G^{a} G_{a} .
$$


The flatness condition of vanishing cosmological constant is that $V=0$ on the vacuum, and it implies:

$$
-3+G^{r} G_{r}+\frac{1}{2} e^{-G} G^{a} G_{a}=0 .
$$

The stationarity conditions correspond to requiring that $\nabla_{r} V=0$, and read:

$$
G_{r}+G^{s} \nabla_{r} G_{s}+e^{-G}\left[G_{a r} G^{a}+\frac{1}{2}\left(h_{a b r}-h_{a b} G_{r}\right) G^{a} G^{b}\right]=0 .
$$

The Hermitian block of the mass matrix for small fluctuations of the scalar fields around the vacuum can be computed as $m_{r \bar{s}}^{2}=\nabla_{r} \nabla_{\bar{s}} V$, and is found to be $[32,33,12]$ :

$$
\begin{aligned}
m_{r \bar{s}}^{2}=e^{G} & {\left[g_{r \bar{s}}+\nabla_{r} G_{t} \nabla_{\bar{s}} G^{t}-R_{r \bar{s} t \bar{u}} G^{t} G^{\bar{u}}\right] } \\
+ & {\left[h^{a b} G_{a r} G_{b \bar{s}}+\left(G_{a r \bar{s}}-2 h^{b c} h_{a b(r} G_{c \bar{s})}-2 G_{a(r} G_{\bar{s})}\right) G^{a}\right.} \\
& \left.-\frac{1}{2}\left(h_{a b} g_{r \bar{s}}-2 h^{c d} h_{a c r} h_{b d \bar{s}}-2 h_{a b(r} G_{\bar{s})}-h_{a b} G_{r} G_{\bar{s}}\right) G^{a} G^{b}\right] .
\end{aligned}
$$

The symmetric mass matrix for the vector fields has the form:

$$
M_{a b}^{2}=2 g^{r \bar{s}} G_{a r} G_{b \bar{s}} .
$$

Finally, the gravitino mass is given by $m_{3 / 2}=e^{G / 2}$.

The auxiliary fields controlling supersymmetry breaking are determined by their equations of motion and are given by

$$
F_{r}=-e^{G / 2} G_{r}, \quad D_{a}=-G_{a} .
$$

These fields are however not independent from each other. A first kinematical relation between them, which holds at any point of field space and represents a constraint due to gravity, is implied by the gauge invariance of the function $G$. It reads:

$$
D_{a}=-i \frac{X_{a}^{r}}{m_{3 / 2}} F_{r}
$$

A second dynamical relation between them, which holds only at stationary points of $V$ and exists independently of gravity, comes from the stationarity conditions along the complex partners of the would-be Goldstone directions. Indeed, contracting the stationarity conditions with $X_{a}^{r}$ and taking the imaginary part, one deduces that [34] (see also [26, 12]):

$$
G_{a r \bar{s}} F^{r} F^{\bar{s}}+\frac{1}{2}\left[M_{a b}^{2}+2\left(F^{r} F_{r}-m_{3 / 2}^{2}\right) h_{a b}\right] D^{b}-\frac{1}{2} Q_{a b c} D^{b} D^{c}=0 .
$$

Let us now subdivide the chiral multiplets $Z^{r}$ into visible sector multiplets $Q^{\alpha}$ and hidden sector multiplets $X^{i}$. The visible sector multiplets $Q^{\alpha}$ are distinguished by the fact that all their components have vanishing VEV. This implies in particular that $G_{\alpha}=0$, $g_{\alpha \vec{\imath}}=0$ and $\nabla_{\alpha} G_{i}=\nabla_{i} G_{\alpha}=0$ on the vacuum. We will furthermore focus on matter fields in chiral representations of the visible gauge group that do not admit holomorphic 
quadratic invariants. This implies that $\nabla_{\alpha} G_{\beta}=0$. We will also require that extra gauge symmetries are at most linearly realized on them, so that $G_{a \alpha}=0$ on the vacuum. Finally, since the gauge kinetic function must be invariant under the visible gauge group, one also has $h_{a b \alpha}=0$. For the hidden sector multiplets $X^{i}$, on the other hand, we do not impose any particular constraint for the moment.

The hidden sector fields can have a pretty generic dynamics. There are however two strong requirements that must be imposed in order to get a satisfactory situation at a certain stationary point. The first is the flatness condition of vanishing vacuum energy, and implies a restriction on the length of the Goldstino vector:

$$
g_{i \vec{\jmath}} F^{i} F^{\vec{\jmath}}=3 m_{3 / 2}^{2}-\frac{1}{2} D^{a} D_{a} .
$$

The second is the stability condition of positivity of the mass matrix for small fluctuations around the vacuum. As shown in $[10,11,12]$, a necessary condition for this to happen is that the Hermitian block $m_{i j}^{2}$ be positive along the direction $G^{i}$, implying the constraint $m_{i \bar{j}}^{2} G^{i} G^{\bar{j}}>0$. This leads to the following condition, which restricts the direction of the Goldstino vector [12]:

$$
\begin{aligned}
R_{i \vec{\jmath} p \bar{q}} F^{i} F^{\vec{\jmath}} F^{p} F^{\bar{q}} \leq & 6 m_{3 / 2}^{4}+\left(M_{a b}^{2}-2 m_{3 / 2}^{2} h_{a b}+h^{c d} h_{a c i} h_{b d \bar{\jmath}} F^{i} F^{\bar{\jmath}}\right) D^{a} D^{b} \\
& +\frac{3}{4} m_{3 / 2} Q_{a b c} D^{a} D^{b} D^{c}-\frac{1}{2}\left(h_{a b} h_{c d}-\frac{1}{2} h_{a b}{ }^{k} h_{c d k}\right) D^{a} D^{b} D^{c} D^{d} .
\end{aligned}
$$

The additional vector fields have a mass matrix which is automatically positive definite and by assumption entirely generated by the hidden sector fields. It takes the form:

$$
M_{a b}^{2}=2 g^{i \vec{\jmath}} G_{a i} G_{b \vec{\jmath}} .
$$

The visible sector fields get soft masses through higher-dimensional operators mixing them to the hidden sector fields. Under the assumptions made above, the non-trivial Hermitian block of the scalar mass matrix takes the following form:

$$
m_{\alpha \bar{\beta}}^{2}=-\left(R_{\alpha \bar{\beta} p \bar{q}}-\frac{1}{3} g_{\alpha \bar{\beta}} g_{p \bar{q}}\right) F^{p} F^{\bar{q}}-\left(G_{a \alpha \bar{\beta}}-\frac{1}{3} g_{\alpha \bar{\beta}} G_{a}\right) D^{a} .
$$

One can rewrite this expression in an alternative form, which uses the parametrization $G=-3 \ln (-\Omega / 3)$ that is naturally suggested by the superconformal approach. With the same assumptions as before, one can easily verify that at the vacuum the following relations hold true:

$$
\begin{aligned}
& R_{\alpha \bar{\beta} p \bar{q}}-\frac{1}{3} g_{\alpha \bar{\beta}} g_{p \bar{q}}=-\frac{3}{\Omega}\left(\Omega_{\alpha \bar{\beta} p \bar{q}}-\Omega^{-1 \gamma \bar{\delta}} \Omega_{\alpha p \bar{\delta}} \Omega_{\bar{\beta} \bar{q} \gamma}\right) \\
& G_{a \alpha \bar{\beta}}-\frac{1}{3} g_{\alpha \bar{\beta}} G_{a}=-\frac{3}{\Omega} \Omega_{a \alpha \bar{\beta}} .
\end{aligned}
$$

Plugging these expressions into eq. (2.12) one finds:

$$
m_{\alpha \bar{\beta}}^{2}=\frac{3}{\Omega}\left[\left(\Omega_{\alpha \bar{\beta} p \bar{q}}-\Omega^{-1 \gamma \bar{\delta}} \Omega_{\alpha p \bar{\delta}} \Omega_{\bar{\beta} \bar{q} \gamma}\right) F^{p} F^{\bar{q}}+\Omega_{a \alpha \bar{\beta}} D^{a}\right] .
$$


This expression can also be obtained directly from the superconformal formulation. The auxiliary field $F$ of the compensator chiral multiplet does not give any contribution ${ }^{1}$. The auxiliary fields $F^{i}$ of the hidden sector chiral multiplets give the first term directly and the second through their mixing with the auxiliary fields $F^{\alpha}$ of the visible sector chiral multiplets. The auxiliary fields $D^{a}$ of the vector multiplets give the last term. Finally, the overall factor is due to the Weyl rescaling associated to the gauge choice $\phi=e^{G / 6}$.

The two equivalent expressions (2.12) and (2.15) for the soft masses are both useful, in different respects. The form (2.12) exhibits an interpretation of the masses in terms of Kähler geometry. It shows for instance that the $F$-term contribution vanishes for maximally symmetric spaces of constant curvature scale equal to $2 / 3$, corresponding to "no-scale" models [35], for which $R_{\alpha \bar{\beta} p \bar{q}}=1 / 3 g_{\alpha \bar{\beta}} g_{p \bar{q}}$, and similary that the $D$-term contribution vanishes whenever $G_{a i \bar{\jmath}}=1 / 3 g_{i \bar{\jmath}} G_{a}$. The form (2.12) gives instead an interpretation of the masses that is more physically connected to the rigid limit intuition. It shows that the $F$-term contribution vanishes whenever the function $\Omega$ is separable into two distinct parts describing the visible and the hidden sectors, corresponding to the so-called sequestered situation [36], and similarly that the $D$-term contribution vanishes whenever the function $\Omega$ does not contain any minimal coupling between the visible sector fields and the vector fields.

\section{$3 \quad$ Effects of heavy vector multiplets}

The effect of vector multiplets relative to that of chiral multiplets substantially simplifies whenever the mass eigenvalues of the vector fields are all much larger than the gravitino mass: $M_{a} \gg m_{3 / 2}$. We also assume that $M_{a} \lesssim 1$, since the vectors are kept in the effective theory below the Planck scale, and that $m_{3 / 2} \ll 1$, in order for supersymmetry to help explaining the hierarchy between the Fermi and the Planck scale. The above conditions for the vector masses are usually verified for the vector fields associated to anomalous $U(1)$ symmetries with a Green-Schwarz mechanism in string models. We will therefore focus on this situation of heavy vector fields from now on, and proceed to derive some simple general results emerging in this limit. Most importantly, it turns out that the vector auxiliary fields $D^{a}$ become small compared to the chiral auxiliary fields $F^{i}$, and the vector multiplet dynamics decouples from the supersymmetry breaking dynamics, which is dominated by chiral multiplets, leaving only simple corrections. These corrections are formally sub-leading, but they can nevertheless be relevant.

In the limit $M_{a} \gg m_{3 / 2}$, the flatness condition (2.2) and the dynamical relation (2.8)

\footnotetext{
${ }^{1}$ Note that this is a consequence of the vanishing of the cosmological constant. In the presence of a cosmological constant $V$, one would find a contribution $-2 \Omega_{\alpha \bar{\beta}} / \Omega V$ or equivalently $2 / 3 g_{\alpha \bar{\beta}} V$, which is entirely due to the Weyl rescaling of the potential by the factor $|\phi|^{4}$, which for the Einstein frame is equal to $e^{2 G / 3}$.
} 
imply together that $F^{i} \sim \mathcal{O}\left(m_{3 / 2}\right)$ and $D^{a} \sim \mathcal{O}\left(m_{3 / 2}^{2} / M_{a}^{2}\right)$, with:

$$
D^{a} \simeq-2 M^{-2 a b} G_{b i \vec{\jmath}} F^{i} F^{\vec{j}} .
$$

The kinematical relation (2.7) implies then that the VEV of the scalar fields arrange in such a way that their contributions to $D^{a}$ cancel each other at the leading order $\mathcal{O}\left(m_{3 / 2}\right)$, and leave only a subleading effect of order $\mathcal{O}\left(m_{3 / 2}^{2} / M_{a}^{2}\right)$ as implied by (3.1). In other words, we get following the approximate $D$-flatness conditions:

$$
X_{a}^{i} F_{i} \simeq 0
$$

In this situation, the flatness and stability constraints on the Goldstino vector of auxiliary fields can be approximated as:

$$
F^{i} F_{i} \simeq 3 m_{3 / 2}^{2}
$$

and

$$
R_{i \bar{\jmath} p \bar{q}} F^{i} F^{\vec{\jmath}} F^{p} F^{\bar{q}}-M_{a b}^{2} D^{a} D^{b} \lesssim 6 m_{3 / 2}^{4} .
$$

The soft masses can in turn be rewritten in the form:

$$
m_{\alpha \bar{\beta}}^{2} \simeq g_{\alpha \bar{\beta}} m_{3 / 2}^{2}-R_{\alpha \bar{\beta} p \bar{q}} F^{p} F^{\bar{q}}-G_{a \alpha \bar{\beta}} D^{a} .
$$

Whenever the vector fields are much heavier that the gravitino, as in the above situation, one can actually account for their leading-order effect in a much simpler way. Indeed, the scale of gauge symmetry breaking is then much higher than the scale of supersymmetry breaking. One can then first integrate out the heavy vector multiplets to define a simpler effective theory with only chiral multiplets, which can be used to describe supersymmetry breaking. The vector multiplets can be integrated out directly at the level of superfields. In doing so, one can neglect terms with supercovariant derivatives, and freeze the superconformal compensator to $\Phi=e^{G / 6}$. The relevant equation of motion of the vector superfield in terms of the chiral superfield is then simply that $G_{a} \simeq 0$. One must then choose a supersymmetric gauge. It is convenient to impose for this the gauge-fixing condition $G_{a} \simeq 0$, where the dependence on the vector superfields is now discarded, which corresponds to an approximate unitary gauge. This allows to consistently assume that the superfields $V^{a}$ are small and to make an expansion of the action in powers of $V^{a}$, to linearize the problem. At leading order in $V^{a}$, the equations of motion yields then [12]:

$$
V^{a} \simeq-2 M^{-2 a b} G_{b}
$$

Taking the $D$ component of this expression, where $M_{a b}^{2}$ is treated as a number and $G_{b}$ is taken to depend only the chiral superfields, one recovers then the dynamical relation (3.1). One can also plug back the solution (3.6) into the quadratic approximation to the $V^{a}$-dependent terms in $G$, namely $\Delta G=G_{a} V^{a}+M_{a b}^{2} / 4 V^{a} V^{b}$, to deduce the form of the 
leading order correction that is induced by the heavy vector fields on the dynamics of the light chiral multiplets:

$$
\Delta G \simeq-M^{-2 a b} G_{a} G_{b}
$$

It is straightforward to compute what is the corresponding correction to the Kähler curvature tensor. One finds:

$$
\begin{aligned}
& \Delta R_{i \bar{\jmath} p \bar{q}} \simeq-2 M^{-2 a b}\left(G_{a i \bar{\jmath}} G_{b p \bar{q}}+G_{a i \bar{q}} G_{b p \bar{\jmath}}\right), \\
& \Delta R_{\alpha \bar{\beta} i \bar{\jmath}} \simeq-2 M^{-2 a b} G_{a \alpha \bar{\beta}} G_{b i \bar{\jmath}} .
\end{aligned}
$$

Using these expressions, as well as the relation (3.1), we can then interpret the leading corrections depending on $D^{a}$ in eqs. (3.4) and (3.5) as emerging from the corrections to the curvature tensors in the terms depending on $F^{i}$.

It is now important to observe that the corrections induced by the heavy vector multiplets in eqs. (3.4) and (3.5) are in principle both significant. In fact, we will now see in somewhat more detail that in string models, where the squared masses $M_{a b}^{2}$ are induced by Higgs fields and the symmetry breaking scale is around the Planck scale, that is $M_{a b}^{2} \sim \mathcal{O}(1)$, all the terms in eqs. (3.4) and (3.5) are of the same order of magnitude, as far as the scaling with dimensionfull quantities is concerned. The only particularity of the terms induced through the vector multiplets is that they are proportional to the charges of the involved fields. These can happen to be small, if they reflect a non-minimal coupling induced at a subleading order in perturbation theory.

\section{Models with canonical Higgs fields}

Let us consider first the case of $U(1)$ symmetries that are realized through rephasings on the matter fields $Q^{\alpha}$ and some gauge symmetry breaking fields $H^{x}$ with charges $q_{\alpha a}$ and $q_{x a}$, and through shifts $\delta_{i a}$ on the supersymmetry breaking fields $M^{i}$. This means that the Killing vectors have components that are given by $X_{a}^{\alpha}=i q_{\alpha a} Q^{\alpha}, X_{a}^{x}=i q_{x a} H^{x}$ and $X_{a}^{i}=-i \delta_{i a}$. For simplicity, we shall assume that $\delta_{i a} \ll 1$, as it turns out to be in most of the known string models, and evaluate all the formulae at leading order in $\delta_{i a}$. In such a situation, the would-be goldstone bosons that are absorbed by the gauge vectors are essentially linear combinations of the fields $H^{x}$, with only a small admixture of the fields $M^{i}$. We will consider the minimal situation where the number of gauge symmetry breaking fields $H^{x}$ with non-zero charges $q_{x a}$ is equal to the number of $U(1)$ vector fields $V^{a}$. The charge matrix $q_{x a}$ is then a square matrix that can be inverted, the inverse charge matrix $q_{a x}^{-1}$ being defined to satisfy the identities ${ }^{2} \sum_{a} q_{x a}^{-1} q_{a y}=\delta_{x y}$ and $\sum_{x} q_{a x} q_{x b}^{-1}=\delta_{a b}$. We emphasize here that the shifts $\delta_{i a}$ can be pretty arbitrary. The only restriction that we shall impose is that they are not all zero, the non-vanishing ones setting

\footnotetext{
${ }^{2}$ In this section, we shall make sums over indices of type $a$ and $x$ explicit, to avoid confusions.
} 
the gauge symmetry breaking scales. This means that there can be an arbitrary but nonzero number of anomalous $U(1)$ symmetries and an arbitrary number of non-anomalous $U(1)$ symmetries.

For concreteness, let us take as starting point the following general form of the Kähler potential defining the effective supergravity Lagrangian, with a canonical term for the Higgs fields:

$$
\begin{aligned}
K= & \hat{K}\left(M^{i}+\bar{M}^{i}-\sum_{a} \delta_{i a} V^{a}\right)+\sum_{x} \tilde{K}_{x \bar{x}}\left(M^{i}+\bar{M}^{i}-\sum_{a} \delta_{i a} V^{a}\right) \bar{H}^{x} e^{\sum_{a} q_{x a} V^{a}} H^{x} \\
& +\sum_{\alpha} K_{\alpha \bar{\alpha}}\left(M^{i}+\bar{M}^{i}-\sum_{a} \delta_{i a} V^{a}\right) \bar{Q}^{\alpha} e^{\sum_{a} q_{\alpha a} V^{a}} Q^{\alpha}+\ldots
\end{aligned}
$$

The superpotential is instead left to be a generic gauge-invariant holomorphic function of the shifting moduli $M^{i}$ and the charged Higgs fields $H^{x}$ :

$$
W=\hat{W}\left(M^{i}+\sum_{a, x} \delta_{i a} q_{a x}^{-1} \ln H^{x}\right)+\ldots
$$

The dots in the above expressions denote possible additional terms that are of higher order in the fields $Q^{\alpha}$, whose VEV are vanishing by assumption, and in the fields $H^{x}$, whose VEV will turn out to be small.

Our aim is to compute the total contribution to soft scalar masses of the chiral multiplets of the visible sector, due to both the $F^{i}, F^{x}$ and the $D^{a}$ auxiliary fields of chiral and vector multiplets of the hidden sector. This computation was first done in $[22,23]$, in the context of heterotic orbifold models with a single anomalous $U(1)$, and generalized to type I models with several $U(1)$ 's in [24]. In the following, we shall present a more general computation, which is valid for an arbitrary number of anomalous and non-anomalous $U(1)$ 's, an arbitrary number of shifting and non-shifting moduli, and a generic supersymmetry breaking dynamics compatible with gauge invariance. We will first do the computation along the lines of $[22,23,24]$, but paying attention to the consistency constraints put by gauge invariance of the superpotential. We will then also show how the same result can be obtained in a simpler way by integrating out the heavy vector multiplets directly at the superfield level, along the lines of $[25,26]$.

On the vacuum, and at leading order in $\delta_{i a}$, the non-trivial components of the Kähler metric in the visible and hidden sectors are given by:

$$
g_{\alpha \bar{\alpha}}=K_{\alpha \bar{\alpha}} ; \quad g_{i \vec{\jmath}}=\hat{K}_{i \vec{\jmath}}+\sum_{x} \tilde{K}_{x \bar{x} i \vec{\jmath}}\left|H^{x}\right|^{2}, \quad g_{x \bar{x}}=\tilde{K}_{x \bar{x}}, \quad g_{x \vec{\imath}}=\tilde{K}_{x \overline{x \imath}} \bar{H}^{x} .
$$

Since the off-diagonal terms are small, it is possible to find simple expressions also for the various blocks of the inverse of the metric, at leading order in $H^{x}$. One finds

$$
g^{\alpha \bar{\alpha}}=g_{\alpha \bar{\alpha}}^{-1} ; \quad g^{i \bar{\jmath}}=g_{i \bar{\jmath}}^{-1}, \quad g^{x \bar{x}}=g_{x \bar{x}}^{-1}, \quad g^{x \bar{\imath}}=-\sum_{k} g_{x \bar{x}}^{-1} g_{k \bar{x}} g_{k \bar{\imath}}^{-1} .
$$

The tensor quantities that enter the formula for the soft masses can be easily evaluated. At leading order in $\delta_{i a}$, one finds $R_{\alpha \bar{\alpha} p \bar{q}}=g_{\alpha \bar{\alpha}} \partial_{p} \partial_{\bar{q}} \ln \left(K_{\alpha \bar{\alpha}}\right)$ and $G_{a \alpha \bar{\alpha}}=g_{\alpha \bar{\alpha}} q_{\alpha a}$. The 
soft masses take therefore the form:

$$
m_{\alpha \bar{\alpha}}^{2}=-g_{\alpha \bar{\alpha}}\left[\partial_{p} \partial_{\bar{q}} \ln \left(K_{\alpha \bar{\alpha}} e^{-\hat{K} / 3}\right) F^{p} F^{\bar{q}}+\sum_{a} q_{\alpha a} D^{a}\right] .
$$

Our main task is now to compute the $D^{a}$ 's in terms of the $F^{i}$ 's, after exploiting the fact that in the minimal situation under consideration, gauge invariance completely fixes the VEV of the Higgs scalar fields $H^{x}$ and the corresponding auxiliary fields $F^{x}$ in terms of the VEV of the moduli fields $M^{i}$ and the corresponding auxiliary fields $F^{i}$.

\subsection{Relation between auxiliary fields}

The vector auxiliary fields can be computed through the relation $D_{a}=-G_{a}$ and take the following form:

$$
D_{a}=\sum_{k} \delta_{k a} \hat{K}_{k}-\sum_{x} q_{x a} g_{x \bar{x}}\left|H^{x}\right|^{2}
$$

The mass matrix $M_{a b}^{2}$ of the vectors fields is given by

$$
M_{a b}^{2}=2 \sum_{i, j} g_{i \bar{\jmath}} \delta_{i a} \delta_{\bar{\jmath} b}+2 \sum_{x} q_{x a} q_{x b} g_{x \bar{x}}\left|H^{x}\right|^{2} .
$$

Assuming that the VEV of the fields $M^{i}$ are of order $\mathcal{O}(1)$, and recalling that the shifts $\delta_{i a}$ are of the order of a loop factor, and thus small but not tiny, we see from the first term in the above expression that one indeed naturally gets the situation $M_{a b}^{2} \gg m_{3 / 2}^{2}$ described in section 3 .

At the stationary point of the potential, the VEV that the $D_{a}$ 's actually develop depend quadratically on the auxiliary fields of all the non-trivially transforming chiral multiplets, and are given by the relation (3.1), which becomes now:

$$
D^{a}=-2 \sum_{b} M^{-2 a b}\left(G_{b i j} F^{i} F^{\bar{\jmath}}+G_{b x \bar{x}} F^{x} F^{\bar{x}}+G_{b i \bar{x}} F^{i} F^{\bar{x}}+G_{b x \bar{\jmath}} F^{x} F^{\bar{\jmath}}\right) .
$$

Neglecting terms of order $\mathcal{O}\left(\delta_{i a}\left|H^{x}\right|^{2}\right)$, the coefficients appearing in this expression are found to be $G_{b i \bar{\jmath}}=\sum_{x} q_{x b} \tilde{K}_{x \bar{x} i \bar{\jmath}}\left|H^{x}\right|^{2}-\sum_{k} \delta_{k b} \hat{K}_{k i \bar{\jmath}}, G_{b x \bar{x}}=q_{x b} \tilde{K}_{x \bar{x}}$ and $G_{b x i}=q_{x b} \tilde{K}_{x \bar{x} i} \bar{H}^{x}$. Substituting these expressions into eq. (4.8), completing the squares and using also the expressions (4.3), one can rewrite the result as:

$$
\begin{aligned}
D^{a}=-2 \sum_{b} M^{-2 a b}[ & \left(\sum_{x} q_{x b}\left|H^{x}\right|^{2}\left(\tilde{K}_{x \bar{x} i \bar{\jmath}}-\tilde{K}_{x \bar{x}}^{-1} \tilde{K}_{x \bar{x} i} \tilde{K}_{x \bar{x} \bar{\jmath}}\right)-\sum_{k} \delta_{k b} \hat{K}_{i \bar{\jmath} k}\right) F^{i} F^{\bar{\jmath}} \\
& \left.+\sum_{x} q_{x b} g_{x \bar{x}} \tilde{F}^{x} \tilde{F}^{\bar{x}}\right]
\end{aligned}
$$

where

$$
\tilde{F}^{x}=F^{x}+g^{x \bar{x}} g_{\bar{x} i} F^{i}
$$


The VEV of the fields $H^{x}$ are related to the VEV of the fields $M^{i}$. Indeed, since the auxiliary fields $D_{a}$ must obtain a small VEV of $\mathcal{O}\left(m_{3 / 2}^{2} / M_{a}^{2}\right)$, the terms of $\mathcal{O}\left(m_{3 / 2}\right)$ in the expression (4.6) must cancel out. This leading order approximate $D$-flatness condition $D_{a} \simeq 0$ implies that $\sum_{x} q_{x a} g_{x \bar{x}}\left|H^{x}\right|^{2} \simeq \sum_{k} \delta_{k a} \hat{K}_{k}$. The VEV of the $H^{x}$ 's are then completely determined in terms of the VEV of the $M^{i}$ 's. Indeed, multiplying this equation by $q_{y a}^{-1}$ and summing then over $a$, one extracts:

$$
\left|H^{x}\right| \simeq g_{x \bar{x}}^{-1 / 2} v_{x}
$$

where

$$
v_{x}=\left(\sum_{k, a} \delta_{k a} q_{a x}^{-1} \hat{K}_{k}\right)^{1 / 2} .
$$

The mass of the vector fields (4.7) is dominated by the second term. The mass matrix and its inverse can then be approximately written as:

$$
M_{a b}^{2} \simeq 2 \sum_{x} q_{x a} q_{x b} v_{x}^{2}, \quad M^{-2 a b} \simeq \frac{1}{2} \sum_{x} q_{a x}^{-1} q_{b x}^{-1} v_{x}^{-2}
$$

The values of the auxiliary fields $F_{x}$ are similarly related to the values of the auxiliary fields $F_{i}$. Indeed, according to eq. (2.7), the $D_{a}$ 's can also be written as linear combinations of the $F_{i}$ 's and $F_{x}$ 's: $D_{a}=-i\left(X_{a}^{x} F_{x}+X_{a}^{k} F_{k}\right) / m_{3 / 2}$, and the approximate $D$-flatness conditions $D_{a} \simeq 0$ imply thus also the approximate relations (3.2), which in our case read $\sum_{x} q_{x a} H^{x} F_{x} \simeq \sum_{k} \delta_{k a} F_{k}$. Multiplying this equation by $q_{y a}^{-1}$, summing over $a$ and using the relation (4.11), one deduces then that the $F_{x}$ are completely determined in terms of the $F_{i}$ :

$$
F_{x}=g_{x \bar{x}}^{1 / 2} v_{x}^{-1} \sum_{k, a} \delta_{k a} q_{a x}^{-1} F_{k}
$$

It is now straightforward to derive the values of the auxiliary fields with upper indices, by using the inverse metric (4.4). Since $F_{x}$ is suppressed by a factor of order $\mathcal{O}\left(H^{x}\right)$ with respect to $F_{i}$, it is enough to keep $\mathcal{O}\left(H^{x}\right)$ terms only in $F^{x}$, and neglect them in $F^{i}$. The non-vanishing off-diagonal component $g^{x \bar{j}}$ is thus relevant only in the computation of $F^{x}$, and after using $g^{x \bar{\jmath}}=-g^{x \bar{x}} g^{i \bar{\jmath}} g_{i \bar{x}}$, one finds $F^{x}=g^{x \bar{x}}\left(F_{\bar{x}}-g^{i \bar{\jmath}} g_{i \bar{x}} F_{\bar{\jmath}}\right)$ and $F^{i}=g^{i \vec{\jmath}} F_{\bar{\jmath}}$. This implies then the simple relation $\tilde{F}^{x}=g^{x \bar{x}} F_{\bar{x}}$. Finally, using the relation (4.14), we see that gauge invariance forces the $\tilde{F}^{x}$ 's to be linear combinations of the $F^{i}$ 's:

$$
\tilde{F}^{x}=g_{x \bar{x}}^{-1 / 2} v_{x}^{-1} \sum_{k, a} \delta_{k a} q_{a x}^{-1} \hat{K}_{k \vec{\jmath}} F^{\bar{\jmath}}
$$

The above results on the relation between the auxiliary fields of the Higgs fields and those of the supersymmetry breaking fields are most conveniently summarized through the relation between $F^{x} / H^{x}$ and $F^{i}$. This can be easily deduced from the already mentioned 
relation $F^{x}=g^{x \bar{x}}\left(F_{\bar{x}}-g^{i \bar{j}} g_{i \bar{x}} F_{\bar{\jmath}}\right)$, after using eqs. (4.11) and (4.14). The result takes the following simple form:

$$
\frac{F^{x}}{H^{x}}=\partial_{i} \ln \left(\tilde{K}_{x \bar{x}}^{-1} \sum_{k, c} \delta_{k c} q_{c x}^{-1} \hat{K}_{k}\right) F^{i} .
$$

We can now come back to the problem of evaluating eq. (4.9). Using the above results (4.11), (4.13) and (4.15) for $\left|H^{x}\right|, M^{-2 a b}$ and $\tilde{F}^{x}$, one obtains:

$$
\begin{aligned}
D^{a}=\sum_{x} q_{a x}^{-1}[ & -\left(\tilde{K}_{x \bar{x}}^{-1} \tilde{K}_{x \bar{x} i \bar{\jmath}}-\tilde{K}_{x \bar{x}}^{-2} \tilde{K}_{x \bar{x} i} \tilde{K}_{x \bar{x} \bar{\jmath}}\right) \\
& \left.+\left(\sum_{k, b} \delta_{k b} q_{b x}^{-1} v_{x}^{-2} \hat{K}_{i \bar{\jmath} k}-\sum_{k, l, b, c} \delta_{k b} q_{b x}^{-1} \delta_{l c} q_{c x}^{-1} v_{x}^{-4} \hat{K}_{k \bar{\jmath}} \hat{K}_{i \bar{l}}\right)\right] F^{i} F^{\vec{\jmath}} .
\end{aligned}
$$

Recalling the expression (4.12) for $v_{x}$, this result can finally be rewritten in the very simple form

$$
D^{a}=\sum_{x} q_{a x}^{-1} \partial_{i} \partial_{\bar{\jmath}} \ln \left(\tilde{K}_{x \bar{x}}^{-1} \sum_{k, c} \delta_{k c} q_{c x}^{-1} \hat{K}_{k}\right) F^{i} F^{\bar{\jmath}}
$$

The above result can in fact be derived in a much simpler way by integrating out the heavy vector fields at the superfield level, along the lines of $[37,38]$ and $[25,26]$. In the limit where $M_{a} \gg m_{3 / 2}$, supercovariant derivatives can be neglected and the equations of motion are entirely controlled by the Kähler potential. To start with, it is convenient to define the following new gauge-invariant combinations of chiral and vector fields:

$$
\begin{aligned}
Q^{\prime \alpha} & =Q^{\alpha} \exp \left\{-\sum_{x, a} q_{\alpha a} q_{a x}^{-1} \ln H^{x}\right\}, \\
M^{\prime i} & =M^{i}+\sum_{x, a} \delta_{i a} q_{a x}^{-1} \ln H^{x}, \\
V^{\prime a} & =V^{a}+\sum_{x} q_{a x}^{-1}\left(\ln H^{x}+\ln \bar{H}^{x}\right) .
\end{aligned}
$$

These allow to rewrite the Kähler potential and the superpotential with one less type of fields, namely without any $H_{x}^{\prime}$. In fact, this procedure can actually be interpreted as a gauge-fixing corresponding to setting $H_{x}^{\prime}=1$, with the choice $\Lambda^{a}=i \sum_{x} q_{a x}^{-1} \ln H^{x}$ for the superfield gauge parameter. The Kähler potential can then be rewritten as

$$
\begin{aligned}
K= & \hat{K}\left(M^{\prime i}+\bar{M}^{\prime i}-\sum_{a} \delta_{i a} V^{\prime a}\right)+\sum_{x} \tilde{K}_{x \bar{x}}\left(M^{\prime i}+\bar{M}^{\prime i}-\sum_{a} \delta_{i a} V^{\prime a}\right) e^{\sum_{a} q_{x a} V^{\prime a}} \\
& +\sum_{\alpha} K_{\alpha \bar{\alpha}}\left(M^{\prime i}+\bar{M}^{\prime i}-\sum_{a} \delta_{i a} V^{\prime a}\right) \bar{Q}^{\prime \alpha} e^{\sum_{a} q_{\alpha a} V^{\prime a}} Q^{\prime \alpha}+\ldots
\end{aligned}
$$

and the superpotential as

$$
W=\hat{W}\left(M^{i}\right)+\ldots
$$


The superfield equations of motion for $V^{\prime a}$ are now easily computed by taking a derivative of $K$ with respect to $V^{\prime a}$. At leading order in $\delta_{i a}$, and ignoring the visible sector fields, one finds:

$$
\sum_{x} q_{x a} \tilde{K}_{x \bar{x}}\left(M^{\prime i}+\bar{M}^{\prime i}\right) e^{\sum_{b} q_{x b} V^{\prime b}}=\sum_{k} \delta_{k a} \hat{K}_{k}\left(M^{\prime i}+\bar{M}^{\prime i}\right) .
$$

Notice that we could neglect the dependence on $V^{\prime a}$ in all the brackets. This is a radical simplification, which is crucial to be able to algebraically solve for $V^{\prime a}$. The solution for $V^{\prime a}$ is finally obtained by using the inverse charge matrix $q_{a x}^{-1}$. One finds:

$$
V^{\prime a}=\sum_{x} q_{a x}^{-1} \ln \left(\tilde{K}_{x \bar{x}}^{-1} \sum_{k, c} \delta_{k c} q_{c x}^{-1} \hat{K}_{k}\right)
$$

Having found the solution of the superfield equation of motion for $V^{\prime a}$, it is now trivial to deduce the value of its $D^{\prime a}$ auxiliary component in terms of the auxiliary fields $F^{\prime i}$. Taking the $D$-component of (4.23), one reproduces indeed the result (4.18):

$$
D^{\prime a}=\sum_{x} q_{a x}^{-1} \partial_{i} \partial_{\bar{\jmath}} \ln \left(\tilde{K}_{x \bar{x}}^{-1} \sum_{k, c} \delta_{k c} q_{c x}^{-1} \hat{K}_{k}\right) F^{\prime i} F^{\prime \bar{\jmath}}
$$

\subsection{Structure of the soft masses}

The soft masses of the visible fields are given by the expressions (4.5) and (4.18), and have the following rather simple and compact structure:

$$
m_{\alpha \bar{\alpha}}^{2}=-g_{\alpha \bar{\alpha}} \partial_{i} \partial_{\bar{j}}\left[\ln \left(K_{\alpha \bar{\alpha}} e^{-\hat{K} / 3}\right)+\sum_{a, x} q_{\alpha a} q_{a x}^{-1} \ln \left(\tilde{K}_{x \bar{x}}^{-1} \sum_{k, c} \delta_{k c} q_{c x}^{-1} \hat{K}_{k}\right)\right] F^{i} F^{\vec{\jmath}}
$$

The expression (4.25) represents the result we were aiming at. It shows that the results of $[25,26]$ generalize in a pretty simple way to more general situations involving several $U(1)$ symmetries, which can be either anomalous or non-anomalous, with equally many gauge symmetry breaking fields, and an arbitrary set of moduli fields, which can either shift or not shift under gauge transformations.

The physical soft masses can be obtained by rescaling the above expression by the metric $g_{\alpha \bar{\alpha}}$, which appears as a non-trivial wave-function factor in the kinetic term. The result can then be rewritten in the following suggestive form:

$$
m_{\alpha}^{2}=m_{F \alpha}^{2}-\sum_{a, x} q_{\alpha a} q_{a x}^{-1}\left(m_{F x}^{2}-m_{A x}^{2}\right)
$$

where

$$
\begin{aligned}
& m_{F \alpha}^{2}=-\partial_{i} \partial_{\bar{\jmath}} \ln \left(K_{\alpha \bar{\alpha}} e^{-\hat{K} / 3}\right) F^{i} F^{\bar{\jmath}} \\
& m_{F x}^{2}=-\partial_{i} \partial_{\bar{\jmath}} \ln \left(\tilde{K}_{x \bar{x}} e^{-\hat{K} / 3}\right) F^{i} F^{\vec{\jmath}} \\
& m_{A x}^{2}=-\partial_{i} \partial_{\bar{\jmath}} \ln \left(\sum_{c} q_{c x}^{-1} \hat{K}_{c} e^{-\hat{K} / 3}\right) F^{i} F^{\vec{\jmath}} .
\end{aligned}
$$


The first term is the usual $F$-term contribution. The second term isolates a general part of the $D$-term contribution that has the same form as for non-anomalous $U(1)$ symmetries, and consists in a linear combination of the F-term masses of the Higgs fields suitably weighted by their charges. Finally, the third contributions encodes essentially the non-trivial effects due to the fact that some $U(1)$ symmetries are anomalous, with some supersymmetry breaking fields transforming non-trivially under them.

One can also compute the form of the flatness and stability constraints (3.3) and (3.4). The effects of chiral multiplets correspond to the unique term in (3.3) and the first term in (3.4). In the case treated here, one should in principle include the effects of both the $M^{i}$ and the $H^{x}$ fields. However, the terms involving $F^{x}$ are negligible with respect to the terms involving $F^{i}$, meaning that that the effect of the $H^{x}$ fields can be neglected. The effects of the fields $V^{a}$ have already been neglected in (3.3) but lead to the potentially sizable second term in (3.4). This term can be computed by using the result (4.18), and can then be reexpressed as a correction to the effective curvature for the fields $M^{i}$. However, it also turns out to be negligible, essentially because the "charges" induced for the moduli fields are only of order $\mathcal{O}\left(\delta_{i a}\right)$ and thus small. In the end, the flatness and stability constraints can be written in terms of the metric and the curvature of the moduli space simply as:

$$
\begin{aligned}
& g_{i \bar{\jmath}} F^{i} F^{\bar{\jmath}} \simeq 3 m_{3 / 2}^{2}, \\
& R_{i \vec{\jmath} p \bar{q}} F^{i} F^{\bar{\jmath}} F^{p} F^{\bar{q}} \lesssim 6 m_{3 / 2}^{4} .
\end{aligned}
$$

The physical components of the Goldstino directions can be obtained by suitably rescaling the auxiliary fields by the square root of the metric, which appears in front of their kinetic terms.

Notice finally that the rescaling procedure needed to switch to canonical scalar and auxiliary fields corresponds geometrically to switching to normal coordinates around the vacuum point. It is then natural to rewrite the above results in this local frame, whose indices will be denoted with capital letters. This is done simply by defining $Q^{\alpha}=e_{\alpha}^{\alpha} Q^{\alpha}$, $M^{I}=e_{i}^{I} M^{i}, H^{X}=e_{x}^{X} H^{x}$, and $V^{A}=e_{a}^{A} V^{a}$, with the help of the vielbeins $e_{\alpha}^{\alpha}, e_{i}^{I}, e_{x}^{X}$ and $e_{a}^{A}$. In this new basis the metrics are trivial: $g_{\alpha \bar{\beta}}=\delta_{\alpha \bar{\beta}}, g_{I \bar{J}}=\delta_{I \bar{J}}, g_{X \bar{Y}}=\delta_{X \bar{Y}}$ and $h_{A B}=\delta_{A B}$. The physical soft scalar masses are then given in terms of the rescaled auxiliary fields $F^{I}=e_{i}^{I} F^{i}$ by

$$
m_{\alpha \bar{\alpha}}^{2}=\left[\frac{1}{3} \delta_{I \bar{J}}-R_{\alpha \bar{\alpha} I \bar{J}}+\sum_{A, X} q_{\alpha A} q_{A X}^{-1}\left(R_{X \bar{X} I \bar{J}}-S_{X \bar{X} I \bar{J}}\right)\right] F^{I} F^{\bar{J}},
$$

and the flatness and stability conditions by

$$
\begin{aligned}
& \delta_{I \bar{J}} F^{I} \bar{F}^{\bar{J}} \simeq 3 m_{3 / 2}^{2}, \\
& R_{I \bar{J} P \bar{Q}} F^{I} \bar{F}^{\bar{J}} F^{P} \bar{F}^{\bar{Q}} \lesssim 6 m_{3 / 2}^{4},
\end{aligned}
$$


where $q_{\alpha A} \equiv q_{\alpha a}, q_{X A} \equiv q_{x a}$ and

$$
\begin{aligned}
& R_{I \bar{J} P \bar{Q}}=e_{I}^{i} e_{\bar{J}}^{\bar{j}} e_{P}^{p} e_{\bar{Q}}^{\bar{q}}\left(\hat{K}_{i \bar{\jmath} p \bar{q}}-\hat{K}^{r \bar{s}} \hat{K}_{i p \bar{s}} \hat{K}_{\bar{\jmath} \bar{q} r}\right), \\
& R_{\alpha \bar{\alpha} I \bar{J}}=e_{I}^{i} e_{\bar{J}}^{\bar{j}} \partial_{i} \partial_{\bar{\jmath}} \ln \left(K_{\alpha \bar{\alpha}}\right), \quad R_{X \bar{X} I \bar{J}}=e_{I}^{i} e_{\bar{J}}^{\bar{j}} \partial_{i} \partial_{\bar{\jmath}} \ln \left(\tilde{K}_{x \bar{x}}\right), \\
& S_{X \bar{X} I \bar{J}}=e_{I}^{i} e_{\bar{J}}^{\bar{j}} \partial_{i} \partial_{\bar{\jmath}} \ln \left(\sum_{k, c} \delta_{k c} q_{c x}^{-1} \hat{K}_{k}\right) .
\end{aligned}
$$

\subsection{Basic moduli}

Let us now illustrate the general result derived above in the particular case where the supersymmetry breaking fields are a set of universal string moduli, each spanning a distinct one-dimensional Kähler manifold, which in the low-energy limit has the form of an $S U(1,1) / U(1)$ coset space. In general, all these moduli can shift under the $U(1)$ symmetries, with arbitrary but small shifts $\delta_{i a}$. The Kähler potential has then the following structure:

$$
\hat{K}=-\sum_{i} c_{i} \ln \left(M^{i}+\bar{M}^{i}-\sum_{a} \delta_{i a} V^{a}\right)
$$

Applying eqs. (4.33), one easily finds:

$$
\begin{aligned}
& R_{I \bar{J} P \bar{Q}}=\frac{2}{c_{I}} \delta_{I \bar{J} P \bar{Q}}, \\
& S_{X \bar{X} I \bar{J}}=\frac{2}{c_{I}} \frac{t_{I X}}{\left(\sum_{K} t_{K X}\right)} \delta_{I \bar{J}}-\frac{1}{\sqrt{c_{I} c_{J}}} \frac{t_{I X} t_{J X}}{\left(\sum_{K} t_{K X}\right)^{2}},
\end{aligned}
$$

in terms of the following quantities, proportional to the inverse of the VEV of the moduli fields through a particular linear combination of their shift parameters:

$$
t_{I X}=\sum_{a} \delta_{i a} q_{a x}^{-1} c_{i}\left(M^{i}+\bar{M}^{i}\right)^{-1}
$$

The quantities $R_{\alpha \bar{\alpha} I \bar{J}}$ and $R_{X \bar{X} I \bar{J}}$ represent the unspecified mixed components of the curvature corresponding to the direct mixing between matter and Higgs fields with moduli fields. These generically depend on the VEV of the moduli fields, but there exist also special situation where they are constant. For instance, if $K_{\alpha \bar{\alpha}}=\prod_{i}\left(M^{i}+\bar{M}^{i}-\sum_{a} \delta_{i a} V^{a}\right)^{n_{\alpha i}}$ and $K_{x \bar{x}}=\prod_{i}\left(M^{i}+\bar{M}^{i}-\sum_{a} \delta_{i a} V^{a}\right)^{n_{x i}}$, with constant modular weights $n_{\alpha i}$ and $n_{x i}$, like in modular-invariant heterotic models, then one finds simply $R_{\alpha \bar{\alpha} I \bar{J}}=-n_{\alpha I} \delta_{I \bar{J}}$ and $R_{X \bar{X} I \bar{J}}=-n_{X I} \delta_{I \bar{J}}$. On the other hand, the quantity $S_{X \bar{X} I \bar{J}}$ has a fixed dependence on the moduli fields, which is non-trivial as soon as several fields shift under $U(1)$ symmetries. In the particular case of models with a single anomalous $U(1)$ and a single shifting modulus $M^{0}$, one has however $t_{I X}=t_{0 X} \delta_{0 I}$ and one finds thus a constant result given by $S_{X \bar{X} I \bar{J}}=c_{0}^{-1} \delta_{0 I \bar{J}}$, independently of the values of the shift and the charges. 


\section{Models with non-canonical Higgs fields}

Let us consider next the case of $U(1)$ symmetries that are realized through rephasings on the matter fields $Q^{\alpha}$ and through shifts $\eta_{x a}$ and $\delta_{i a}$ on the gauge symmetry breaking fields $M^{x}$ and on the supersymmetry breaking fields $M^{i}$. This means that the components of the Killing vectors defining the action of the $U(1)$ symmetries are given by $X_{a}^{\alpha}=i q_{\alpha a} Q^{\alpha}$, $X_{a}^{x}=-i \eta_{x a}$ and $X_{a}^{i}=-i \delta_{i a}$. For simplicity, we shall again assume for the moment that $\delta_{i a} \ll 1$, and evaluate all the formulae at leading order in the parameters $\delta_{i a}$. But we will instead work exactly in the quantities $\eta_{x a}$. In this situation, the would-be goldstone bosons that are absorbed by the gauge vectors are mostly linear combinations of the fields $M^{x}$, with only a small mixture of the fields $M^{i}$. We will also consider again the minimal situation where the number of gauge symmetry breaking fields $M^{x}$ with non-zero shifts $\eta_{x a}$ is equal to the number of $U(1)$ vector fields $V^{a}$. The shift matrix $\eta_{x a}$ is then a square matrix that admits an inverse $\eta_{a x}^{-1}$.

In this case, we will take as starting point the following general form of the Kähler potential, with an unspecified non-canonical term for the Higgs fields:

$$
\begin{aligned}
K= & \hat{K}\left(M^{i}+\bar{M}^{i}-\sum_{a} \delta_{i a} V^{a}\right)+\tilde{K}\left(M^{x}+\bar{M}^{x}-\sum_{a} \eta_{x a} V^{a}\right) \\
& +\sum_{\alpha} K_{\alpha \bar{\alpha}}\left(M^{i}+\bar{M}^{i}-\sum_{a} \delta_{i a} V^{a}\right) \bar{Q}^{\alpha} e^{\sum_{a} q_{\alpha a} V^{a}} Q^{\alpha}+\ldots
\end{aligned}
$$

The superpotential is instead left to be a generic gauge-invariant holomorphic function of the shifting moduli $M^{i}$ and $M^{x}$ :

$$
W=\hat{W}\left(M^{i}-\sum_{a, x} \delta_{i a} \eta_{a x}^{-1} M^{x}\right)+\ldots
$$

The dots denote as before possible additional terms which are of higher order in the fields and can therefore be neglected.

On the vacuum, the Kähler metric is in this case block-diagonal, and the soft masses take the same form as before:

$$
m_{\alpha \bar{\alpha}}^{2}=-g_{\alpha \bar{\alpha}}\left[\partial_{p} \partial_{\bar{q}} \ln \left(K_{\alpha \bar{\alpha}} e^{-\hat{K} / 3}\right) F^{p} F^{\bar{q}}+\sum_{a} q_{\alpha a} D^{a}\right] .
$$

Our main task is again to compute the $D^{a}$ 's in terms of the $F^{i}$ 's, after exploiting the fact that in the minimal situation under consideration, gauge invariance completely fixes the $\mathrm{VEV}$ of $M^{x}$ and $F^{x}$ in terms of the VEV of $M^{i}$ and $F^{i}$.

\subsection{Relation between auxiliary fields}

The vector auxiliary fields is determined by the relation $D_{a}=-G_{a}$ and takes in this case the following form:

$$
D_{a}=\sum_{k} \delta_{k a} \hat{K}_{k}+\sum_{x} \eta_{x a} \tilde{K}_{x}
$$


The mass matrix $M_{a b}^{2}$ of the vectors fields is given by

$$
M_{a b}^{2}=2 \sum_{i, j} \delta_{i a} \delta_{\bar{\jmath} b} \hat{K}_{i \vec{\jmath}}+2 \sum_{x, y} \eta_{x a} \eta_{y b} \tilde{K}_{x y}
$$

At the stationary point of the potential, the VEV that the $D_{a}$ actually develop are given by the relation (3.1):

$$
D^{a}=-2 \sum_{b} M^{-2 a b}\left(G_{b i \bar{\jmath}} F^{i} F^{\bar{\jmath}}+G_{b x \bar{y}} F^{x} F^{\bar{y}}\right) .
$$

One easily calculates $G_{b i \bar{\jmath}}=-\sum_{k} \delta_{k b} \hat{K}_{k i \bar{\jmath}}$ and $G_{b x \bar{y}}=-\sum_{z} \eta_{z b} \tilde{K}_{z x \bar{y}}$. Substituting these expressions into eq. (5.6), one finds:

$$
D^{a}=2 \sum_{b} M^{-2 a b}\left[\sum_{k} \delta_{k b} \hat{K}_{i \bar{\jmath} k} F^{i} F^{\bar{\jmath}}+\sum_{z} \eta_{z b} \tilde{K}_{x \bar{y} z} F^{x} F^{\bar{y}}\right]
$$

Before proceeding, we shall now make the following further mild assumptions concerning the form of the Kähler potential of the Higgs fields:

$$
\begin{aligned}
& \tilde{K}^{\prime \prime}=\text { diagonal, } \\
& \tilde{K}^{\prime \prime} /\left(\tilde{K}^{\prime}\right)^{2} \gg \hat{K}^{\prime \prime} /\left(\hat{K}^{\prime}\right)^{2} .
\end{aligned}
$$

These assumptions are not strictly necessary, but they simplify the computation enough to be able to carry it out in general.

The VEV of the $M^{x}$ are related to the VEV of the $M^{i}$. Indeed, the approximate $D$-flatness conditions $D_{a} \simeq 0$ imply that $\sum_{x} \eta_{x a} \tilde{K}_{x} \simeq-\sum_{k} \delta_{k a} \hat{K}_{k}$. Thanks to (5.8), one can then formally extract the value of the Higgs fields in terms of the inverse function $\tilde{K}_{x}^{\text {inv }}$ of the function $\tilde{K}_{x}$. One finds:

$$
M^{x}+\bar{M}^{x} \simeq \tilde{K}_{x}^{\mathrm{inv}}\left(v_{x}^{2}\right)
$$

where now

$$
v_{x}=\left(-\sum_{k, a} \delta_{k a} \eta_{a x}^{-1} \hat{K}_{k}\right)^{1 / 2} .
$$

As a consequence of (5.9), the mass of the vector fields (5.5) is again dominated by the second term. The mass matrix and its inverse can then be approximately written as:

$$
M_{a b}^{2} \simeq 2 \sum_{x} \eta_{x a} \eta_{x b} \tilde{K}_{x \bar{x}}, \quad M^{-2 a b} \simeq \frac{1}{2} \sum_{x} \eta_{a x}^{-1} \eta_{b x}^{-1} \tilde{K}_{x \bar{x}}^{-1}
$$

The values of the auxiliary fields $F_{x}$ are similarly related to the values of the auxiliary fields $F_{i}$. Indeed, the approximate relation (3.2) implies that $\sum_{x} \eta_{x a} F_{x} \simeq-\sum_{k} \delta_{k a} F_{k}$. One deduces then that:

$$
F_{x}=-\sum_{k, a} \delta_{k a} \eta_{a x}^{-1} F_{k}
$$


In this case, it is trivial to derive the values of the auxiliary fields with upper indices, since the fields $M^{x}$ and $M^{i}$ do not mix in the Kähler potential. One simply has $F^{x}=g^{x \bar{x}} F_{\bar{x}}$ and $F^{i}=g^{i \vec{\jmath}} F_{\bar{\jmath}}$. This implies then that

$$
F^{x}=-\tilde{K}_{x \bar{x}}^{-1} \sum_{k, a} \delta_{k a} \eta_{a x}^{-1} \hat{K}_{k \bar{\jmath}} F^{\vec{\jmath}}
$$

Finally, the above expression can also be rewritten in a more illuminating form. Indeed, by the definition of the inverse function appearing in $(5.10)$ one has $\left(\tilde{K}_{x}^{\text {inv }}\right)^{\prime}=\tilde{K}_{x \bar{x}}^{-1}$, and thus:

$$
F^{x}=\partial_{i} \tilde{K}_{x}^{\mathrm{inv}}\left(-\sum_{k, c} \delta_{k c} \eta_{c x}^{-1} \hat{K}_{k}\right) F^{i}
$$

We can now evaluate eq. (5.7). Using the above results (5.10), (5.12) and (5.14) for $M^{x}+\bar{M}^{x}, M^{-2 a b}$ and $F^{x}$, one obtains:

$$
D^{a}=\sum_{x} \eta_{a x}^{-1}\left[\sum_{k, b} \delta_{k b} \eta_{b x}^{-1} \tilde{K}_{x \bar{x}}^{-1} \hat{K}_{i \bar{\jmath} k}+\sum_{k, l, b, c} \delta_{k b} \eta_{b x}^{-1} \delta_{l c} \eta_{c x}^{-1} \tilde{K}_{x \bar{x} x} \tilde{K}_{x \bar{x}}^{-3} \hat{K}_{k \bar{\jmath}} \hat{K}_{i \bar{l}}\right] F^{i} F^{\bar{\jmath}}
$$

Recalling the expression (5.11) for $v_{x}$, and noticing that by the definition of the inverse function appearing in $(5.10)$ one has $\left(\tilde{K}_{x}^{\text {inv }}\right)^{\prime}=\tilde{K}_{x \bar{x}}^{-1}$ and $\left(\tilde{K}_{x}^{\text {inv }}\right)^{\prime \prime}=-\tilde{K}_{x x \bar{x}} \tilde{K}_{x \bar{x}}^{-3}$, this result can finally be rewritten simply as

$$
D^{a}=-\sum_{x} \eta_{a x}^{-1} \partial_{i} \partial_{\vec{\jmath}} \tilde{K}_{x}^{\operatorname{inv}}\left(-\sum_{k, c} \delta_{k c} \eta_{c x}^{-1} \hat{K}_{k}\right) F^{i} F^{\vec{\jmath}}
$$

The above result can again be derived in a much simpler way by integrating out the heavy vector fields at the superfield level. To do so, we define the following new gaugeinvariant combinations of chiral and vector fields:

$$
\begin{aligned}
Q^{\prime \alpha} & =Q^{\alpha} \exp \left\{\sum_{x, a} q_{\alpha a} \eta_{a x}^{-1} M^{x}\right\}, \\
M^{\prime i} & =M^{i}-\sum_{x, a} \delta_{i a} \eta_{a x}^{-1} M^{x}, \\
V^{\prime a} & =V^{a}-\sum_{x} \eta_{a x}^{-1}\left(M^{x}+\bar{M}^{x}\right) .
\end{aligned}
$$

These combinations of fields allow to rewrite the theory with one less type of fields, without any $M^{\prime x}$. This procedure can as before be interpreted as a gauge fixing corresponding to setting $M^{\prime x}=0$, with the choice $\Lambda^{a}=-i \sum_{x} \eta_{a x}^{-1} M^{x}$ for the superfield gauge parameter. The Kähler potential can then be rewritten as

$$
\begin{aligned}
K= & \hat{K}\left(M^{\prime i}+\bar{M}^{\prime i}-\sum_{a} \delta_{i a} V^{\prime a}\right)+\tilde{K}\left(-\sum_{a} \eta_{x a} V^{\prime a}\right) \\
& +\sum_{\alpha} K_{\alpha \bar{\alpha}}\left(M^{\prime i}+\bar{M}^{\prime i}-\sum_{a} \delta_{i a} V^{\prime a}\right) \bar{Q}^{\prime \alpha} e^{\sum_{a} q_{\alpha a} V^{\prime a}} Q^{\prime \alpha}+\ldots
\end{aligned}
$$


and the superpotential as

$$
W=\hat{W}\left(M^{i}\right)+\ldots
$$

The superfield equations of motion for $V^{\prime a}$ are now easily computed by taking a derivative with respect to $V^{\prime a}$. At leading order in the shift parameters $\delta_{i a}$, one finds just:

$$
\sum_{x} \eta_{x a} \tilde{K}_{x}\left(-\sum_{b} \eta_{x b} V^{\prime b}\right)=-\sum_{k} \delta_{k a} \hat{K}_{k}\left(M^{\prime i}+\bar{M}^{\prime i}\right) .
$$

From this one easily extracts the following solution for $\hat{V}^{a}$ :

$$
V^{\prime a}=-\sum_{x} \eta_{a x}^{-1} \tilde{K}_{x}^{\mathrm{inv}}\left(-\sum_{k, c} \delta_{k c} \eta_{c x}^{-1} \hat{K}_{k}\right) .
$$

Taking the $D$-component of (5.22), one finally recovers eq. (5.17):

$$
D^{\prime a}=-\sum_{x} \eta_{a x}^{-1} \partial_{i} \partial_{\tilde{\jmath}} \tilde{K}_{x}^{\mathrm{inv}}\left(-\sum_{k, c} \delta_{k c} \eta_{c x}^{-1} \hat{K}_{k}\right) F^{\prime i} F^{\prime \bar{\jmath}}
$$

\section{$5.2 \quad$ Structure of soft masses}

The soft masses of the visible fields are in this case given by the formulae (5.3) and (5.17), and have the following structure:

$$
m_{\alpha \bar{\alpha}}^{2}=-g_{\alpha \bar{\alpha}} \partial_{i} \partial_{\bar{j}}\left[\ln \left(K_{\alpha \bar{\alpha}} e^{-\hat{K} / 3}\right)-\sum_{a, x} q_{\alpha a} \eta_{a x}^{-1} \tilde{K}_{x}^{\operatorname{inv}}\left(-\sum_{k, c} \delta_{k c} \eta_{c x}^{-1} \hat{K}_{k}\right)\right] F^{i} F^{\bar{\jmath}}
$$

The expression (5.24) represents the generalization of (4.25) to the case where the Higgs fields have an arbitrary Kähler potential but do not directly mix to the moduli fields. Taking $\tilde{K}(x)=e^{x}$, so that $\tilde{K}_{x}^{\text {inv }}(y)=\ln (y)$, one recovers the situation of previous section in the particular case $\tilde{K}_{x x}=1$, after identifying $H^{x}=e^{M^{x}}$ and $q_{x a}=-\eta_{x a}$. Eq. (5.24) shows that in the situation where the Higgs fields have a non-canonical Kähler potential, the $D$-term contribution to soft masses has a functional dependence that is determined by the inverse function of the first derivative of this potential. This can in particular happen to vanish if the functional form of the Kähler potential for the Higgs fields is related to that of the moduli fields.

The physical soft masses, obtained by rescaling the above expression by the metric $g_{\alpha \bar{\alpha}}$, can be rewritten in the form:

$$
m_{\alpha}^{2}=m_{F \alpha}^{2}+\sum_{a, x} q_{\alpha a} \eta_{a x}^{-1}\left(m_{F x}^{2}-m_{A x}^{2}\right)
$$

where now

$$
\begin{aligned}
& m_{F \alpha}^{2}=-\partial_{i} \partial_{\bar{\jmath}} \ln \left(K_{\alpha \bar{\alpha}} e^{-\hat{K} / 3}\right) F^{i} F^{\vec{\jmath}}, \\
& m_{F x}^{2}=-\partial_{i} \partial_{\bar{\jmath}} \tilde{K}_{x}^{\operatorname{inv}}\left(e^{-\hat{K} / 3}\right) F^{i} F^{\bar{\jmath}}, \\
& m_{A x}^{2}=-\partial_{i} \partial_{\bar{\jmath}} \tilde{K}_{x}^{\operatorname{inv}}\left(-\sum_{c} \eta_{c x}^{-1} \hat{K}_{c} e^{-\hat{K} / 3}\right) F^{i} F^{\vec{\jmath}} .
\end{aligned}
$$


The first term is the usual $F$-term contribution. The second term isolates as before a general part of the $D$ term contribution consisting of a linear combination of generalized $F$-term masses of the Higgs fields suitably weighted by their charges. Finally, the third contributions encodes the non-trivial effects related to anomalies.

The flatness and stability constraints (3.3) and (3.4) are as before dominated by the moduli fields. The effects of the Higgs fields and of the vector fields are again small as a consequence of the assumption that the shift vectors $\delta_{i a}$ of the supersymmetry breaking fields are small. In the end, the flatness and stability constraints read thus simply:

$$
\begin{aligned}
& g_{i j} F^{i} F^{\bar{\jmath}} \simeq 3 m_{3 / 2}^{2}, \\
& R_{i \bar{\jmath} p \bar{q}} F^{i} F^{\vec{\jmath}} F^{p} F^{\bar{q}} \lesssim 6 m_{3 / 2}^{4} .
\end{aligned}
$$

As before, the physical components of the Goldstino direction are obtained by rescaling the auxiliary fields by the square root of the metric, and the flatness and stability constraints are unchanged.

In the frame of normal coordinates around the vacuum, corresponding to canonically normalized fields, the physical soft scalar masses can also be written as

$$
m_{\alpha \bar{\alpha}}^{2}=\left[\frac{1}{3} \delta_{I \bar{J}}-R_{\alpha \bar{\alpha} I \bar{J}}+\sum_{A, X} q_{\alpha A} \eta_{A X}^{-1} S_{X \bar{X} I \bar{J}}\right] F^{I} F^{\bar{J}},
$$

and the flatness and stability conditions read

$$
\begin{aligned}
& \delta_{I \bar{J}} F^{I} \bar{F}^{\bar{J}}=3 m_{3 / 2}^{2}, \\
& R_{I \bar{J} P \bar{Q}} F^{I} \bar{F}^{\bar{J}} F^{P} \bar{F}^{\bar{Q}} \leq 6 m_{3 / 2}^{4},
\end{aligned}
$$

where $q_{\alpha A} \equiv q_{\alpha a}, \eta_{X A} \equiv \eta_{x a}$ and

$$
\begin{aligned}
& R_{I \bar{J} P \bar{Q}}=e_{I}^{i} e_{\bar{J}}^{\bar{\jmath}} e_{P}^{p} e_{\bar{Q}}^{\bar{q}}\left(\hat{K}_{i \bar{\jmath} p \bar{q}}-\hat{K}^{r \bar{s}} \hat{K}_{i p \bar{s}} \hat{K}_{\bar{\jmath} \bar{q} r}\right), \\
& R_{\alpha \bar{\alpha} I \bar{J}}=e_{I}^{i} e_{\bar{J}}^{\bar{j}} \partial_{i} \partial_{\bar{\jmath}} \ln \left(K_{\alpha \bar{\alpha}}\right) \\
& S_{X \bar{X} I \bar{J}}=e_{I}^{i} e_{\bar{J}}^{\bar{j}} \partial_{i} \partial_{\bar{\jmath}} \tilde{K}_{x}^{\text {inv }}\left(-\sum_{k, c} \delta_{k c} \eta_{c x}^{-1} \hat{K}_{k}\right) .
\end{aligned}
$$

\subsection{Basic moduli}

Let us finally see what happens in the particular case where the supersymmetry breaking fields and the gauge symmetry breaking fields both belong to a set of universal string moduli, each spanning an $S U(1,1) / U(1)$ manifold. In general, all the supersymmetry breaking and gauge symmetry breaking fields can shift under the $U(1)$ symmetries, with shifts $\delta_{i a}$ and $\eta_{x a}$. The Kähler potential has the following structure:

$$
\begin{aligned}
& \hat{K}=-\sum_{i} c_{i} \ln \left(M^{i}+\bar{M}^{i}-\sum_{a} \delta_{i a} V^{a}\right), \\
& \tilde{K}=-\sum_{x} c_{x} \ln \left(M^{x}+\bar{M}^{x}-\sum_{a} \eta_{x a} V^{a}\right) .
\end{aligned}
$$


In this interesting situation, the approximations done to derive eq. (5.29) are unfortunately not valid. In particular, one has $\tilde{K}^{\prime \prime} /\left(\tilde{K}^{\prime}\right)^{2} \sim \hat{K}^{\prime \prime} /\left(\hat{K}^{\prime}\right)^{2}$, in conflict with (5.9). As a consequence, it is not possible to neglect terms with higher powers of $\delta_{i a}$, and one has to do an algebraically exact computation. This is feasible, thanks to the simple form of the Kähler potential, but unfortunately only a case by case analysis seems to be possible. Notice nevertheless that applying eqs. (5.32), one would find:

$$
\begin{aligned}
& R_{I \bar{J} P \bar{Q}}=\frac{2}{c_{I}} \delta_{I \bar{J} P \bar{Q}} \\
& S_{X \bar{X} I \bar{J}}=-\frac{2 c_{X}}{c_{I}} \frac{t_{I X}}{\left(\sum_{K} t_{K X}\right)^{2}} \delta_{I \bar{J}}+\frac{2 c_{X}}{\sqrt{c_{I} c_{J}}} \frac{t_{I X} t_{J X}}{\left(\sum_{K} t_{K X}\right)^{3}},
\end{aligned}
$$

in terms of the quantities

$$
t_{I X}=-\sum_{a} \delta_{i a} \eta_{a x}^{-1} c_{i}\left(M^{i}+\bar{M}^{i}\right)^{-1}
$$

The quantity $S_{X \bar{X} I \bar{J}}$ has again a fixed dependence on the moduli fields. This is non-trivial when several supersymmetry breaking fields shift under $U(1)$ symmetries. But whenever there is a single shifting modulus $M^{0}$ breaking supersymmetry and being stabilized independently, one has $t_{I X}=t_{0 X} \delta_{0 I}$ and the result vanishes: $S_{X \bar{X} I \bar{J}}=0$. This suggests that in this case there is no $D$-term effect in the soft masses.

For any fixed number of supersymmetry and gauge symmetry breaking fields with Kähler potential given by (5.33) and (5.34), it is possible to compute the exact expression for the $D$-term contribution to soft masses in a rather straightforward way, by using the method of integrating out the vector multiplets at the superfield level. In the presence of a single supersymmetry breaking field $M^{0}$ with non-trivial shifts $\delta_{0 a}$, the equations of motions of the vector superfields imply that $\sum_{a} Y_{x a} V^{\prime a}=M^{\prime 0}+\bar{M}^{\prime 0}$, in terms of the square matrix $Y_{x a}=\delta_{0 a}+\left(c_{0} / c_{x}\right) \sum_{b} \delta_{0 b} \eta_{b x}^{-1} \eta_{x a}$. The solution of this linear system of equations takes then the simple form $V^{\prime a}=\left(\sum_{x} Y_{a x}^{-1}\right)\left(M^{\prime 0}+\bar{M}^{\prime 0}\right)$. This result implies that the corresponding $D^{a}$ vanish, and shows that there is indeed no effect in this particular case. It should also be noted that in this case the VEV of the Higgs scalar fields and their auxiliary partners cannot be neglected and contribute on the same footing as the moduli fields to the vacuum energy and the moduli soft masses, and thus to the flatness and stability conditions. In other words, one must in this case include in the set of multiplets contributing to supersymmetry breaking also the Higgs multiplets, and since the VEV of these multiplets are determined in terms of the VEV of the other moduli multiplets, a special restriction on the Goldstino direction arises.

\section{Toroidal string models}

Let us now study more in detail what happens in the simplest string models, based on a toroidal compactification geometry with some discrete orbifold or orientifold projection, 
under the assumption that the supersymmetry breaking sector is identified with the sector of the untwisted moduli. These include the dilaton $S$, the Kähler class and complex structure moduli $T_{r}$ and $U_{s}$, and the Wilson line moduli $Z_{a}$. The scalar fields of the corresponding chiral multiplets control respectively the string coupling, the size and the shape of the compactification geometry, and the structure of the gauge bundle. The Kähler potential of such moduli is determined, at leading order in the weak-coupling and low-energy expansions, by a simple dimensional reduction of the minimal ten-dimensional supergravity theory. On a torus, the moduli space would be the product of an $S U(1,1) / U(1)$ factor, parametrized by $S$, and an $S O(6,6+r) /(S O(6) \times S O(6+r))$ factor, $r$ being the rank of the gauge group, parametrized by the $T_{r}, U_{s}$ and $Z_{a}$. The discrete projection defining the model preserves the first factor but reduces the second factor to a submanifold. This is in general a coset Kähler manifold which cannot be completely factorized. More precisely, it consists of up to 6 basic dimensions spanning $S U(1,1) / U(1,1)$ submanifolds, associated with the universal Kähler class and complex structure moduli $T_{r}$ and $U_{s}$, and a variable number of additional dimensions, associated with additional model-dependent moduli, which enhanced the product of these basic factors to a larger and more symmetric manifold. For simplicity, we will assume that only the basic "diagonal" moduli associated with the $S U(1,1) / U(1)$ submanifolds participate to supersymmetry breaking, whereas the additional "non-diagonal" moduli enhancing the scalar manifold do not have a relevant breaking effect. This assumption is not expected to represent a severe limitation, because the latter are associated with additional isometries, which suggest that it should be possible to rephrase in an equivalent way effects due to off-diagonal moduli as a effects due to diagonal moduli ${ }^{3}$.

Ignoring for the moment vector fields, the Kähler potential for the moduli sector has the following simple and separable structure, at leading order in the low-energy and weekcoupling expansions $[39,40,41]$ :

$$
\hat{K}=-\ln (S+\bar{S})-\sum_{r} \ln \left(T_{r}+\bar{T}_{r}\right)-\sum_{s} \ln \left(U_{s}+\bar{U}_{s}\right) .
$$

The flatness condition implies that the rescaled auxiliary fields can be parametrized in terms of a Goldstino angle $\theta$, controlling the relative importance of the dilaton $S$ and the geometric moduli $T_{r}$ and $U_{s}$, some spherical parameters $\Theta_{r}$ and $\Theta_{s}$, satisfying the constraint $\sum_{r} \Theta_{r}^{2}+\sum_{r} \Theta_{s}^{2}=1$ and parametrizing the relative importance of the different geometric moduli, as well as some arbitrary phases $\gamma, \gamma_{r}$ and $\gamma_{s}$ (the bars denote flat indices) [8]:

$$
\begin{aligned}
& F^{\bar{S}}=\sqrt{3} e^{i \gamma} \sin \theta m_{3 / 2}, \\
& F^{\bar{T}_{r}}=\sqrt{3} \Theta_{r} e^{i \gamma_{r}} \cos \theta m_{3 / 2}, \quad F^{\bar{U}_{s}}=\sqrt{3} \Theta_{s} e^{i \gamma_{s}} \cos \theta m_{3 / 2} .
\end{aligned}
$$

\footnotetext{
${ }^{3}$ This is certainly true for the masses of the moduli themselves, and the off-diagonal fields do not allow to alleviate the flatness and stability conditions arising for the basic moduli [11]. It is generically expected to hold true also for the soft masses, provided however that the isometry group of the moduli space survives as a global symmetry for the whole scalar manifold, including the matter fields.
} 
The stability condition implies further restrictions on the parameters $\theta$ and $\Theta_{r}$. More precisely, it reads

$$
\sin ^{4} \theta+\left(\sum_{r} \Theta_{r}^{4}+\sum_{s} \Theta_{s}^{4}\right) \cos ^{4} \theta \lesssim \frac{1}{3} .
$$

Notice that the quantity $z=\sum_{r} \Theta_{r}^{4}+\sum_{s} \Theta_{s}^{4}$ is maximal when one of the $\Theta_{r, s}$ is equal to 1 and all the other 0 , and minimal when all the $\Theta_{r, s}$ are equal to $1 / \sqrt{n}$, where $n$ is the total number of geometric moduli. So $z \in[1 / n, 1]$, and small and large values of $z$ correspond respectively to very democratic and antidemocratic distributions of the breaking among the geometric moduli. It is straightforward to see that the condition (6.4) can be satisfied for some $\theta$ only if $z \lesssim 1 / 2$. This means that the breaking must be distributed over the various geometric moduli in a sufficiently democratic way, in order to increase their weight compared to the dilaton in the condition. Thus, one finds the necessary condition

$$
\sum_{r} \Theta_{r}^{4}+\sum_{s} \Theta_{s}^{4} \lesssim \frac{1}{2}
$$

When this condition is satisfied, that is $z \lesssim 1 / 2$, the range of Goldstino angles satisfying the bound is given by $\theta \in\left[\theta_{-}, \theta_{+}\right]$, where $\theta_{ \pm}=\arccos [(1 \pm \sqrt{(1-2 z) / 3}) /(1+z)]^{1 / 2}$. For $z \simeq 1 / 2$, there is only one critical value $\theta_{0} \simeq \arcsin \left(3^{-1 / 2}\right)$. For $z<1 / 2$, there are distinct minimal and maximal values $\theta_{-}$and $\theta_{+}$departing monotonically from the critical value $\theta_{0}$. For $z \simeq 1 / 3$, these reach the values $\theta_{-} \simeq 0$ and $\theta_{+} \simeq \pi / 4$. For smaller $z \ll 1 / 3$, the minimal value stays unchanged, $\theta_{-} \simeq 0$, whereas the maximal value saturates at the the absolute upper bound $\theta_{+} \simeq \arcsin \left(3^{-1 / 4}\right)$. In any case, the Goldstino angle must therefore certainly satisfy the following bound:

$$
0 \leq \sin ^{2} \theta<\frac{1}{\sqrt{3}}
$$

The above results show that none of the moduli is allowed to dominate supersymmetry breaking on its own [10] (see also [42]). In particular, dilaton domination is excluded. On the other hand, it is conceivable that the 3 or more of the geometric moduli may dominate.

In the presence of heavy vector fields associated to broken $U(1)$ symmetries, some or all of the moduli may acquire a small shift transformation law. A small VEV for $D$ is then generated. Its effect on the vacuum energy and the moduli masses can be neglected, and the above results concerning the Goldstino direction remain approximately valid. On the other hand, this small $D$ gives significant effects on the soft terms of all the charged fields, whose form depends on the details of the models.

\subsection{Heterotic models}

Let us first study the case of heterotic orbifold models $[43,44]$. In these models, there can be only one anomalous $U(1)$ symmetry, and the Green-Schwarz mechanism involves only $S$ and not $T_{r}$ or $U_{s}$. The Higgs fields are always charged matter fields $H^{x}$ and have a canonical Kähler potential. 
The situation is of the type discussed in section 4, and the general form of the Kähler potential is given by eq. (4.1). The potential of the moduli has the simple form:

$$
\hat{K}=-\ln \left(S+\bar{S}-\sum_{a} \delta_{a} V^{a}\right)-\sum_{r} \ln \left(T_{r}+\bar{T}_{r}\right)-\sum_{s} \ln \left(U_{s}+\bar{U}_{s}\right) .
$$

The couplings between the moduli fields and the matter and Higgs fields also have in this case a simple structure and depend on constant modular weights $n_{\alpha r}, n_{\alpha s}$ and $n_{x r}, n_{x s}$, representing the charges of the matter field $Q_{\alpha}$ and the Higgs field $H^{x}$ with respect to the $U(1)$ isometry transformations associated to the moduli $T_{r}, U_{s}[45,46]$ :

$$
\begin{aligned}
& K_{\alpha \bar{\alpha}}=\prod_{r}\left(T_{r}+\bar{T}_{r}\right)^{n_{\alpha r}} \prod_{s}\left(U_{s}+\bar{U}_{s}\right)^{n_{\alpha s}}, \\
& K_{x \bar{x}}=\prod_{r}\left(T_{r}+\bar{T}_{r}\right)^{n_{x r}} \prod_{s}\left(U_{s}+\bar{U}_{s}\right)^{n_{\alpha s}} .
\end{aligned}
$$

Notice that the corresponding mixed components of the Riemann tensor are given simply by $R_{\alpha \bar{\alpha} I \bar{J}}=-n_{\alpha i} \delta_{i \bar{\jmath}}$ and $R_{X \bar{X} I \bar{J}}=-n_{x i} \delta_{i \bar{\jmath}}$, and are thus field-independent and diagonal. This leads to a very simple structure for the $F$-term contribution to soft terms [8].

The expression for the physically normalized soft scalar masses can be obtained by applying the general results derived in section 4 . One finds the following very simple result

$$
\frac{m_{\alpha}^{2}}{m_{3 / 2}^{2}}=\left[1+n_{\alpha} \cos ^{2} \theta\right]-\sum_{a, x} q_{\alpha a} q_{a x}^{-1}\left[n_{x} \cos ^{2} \theta+3 \sin ^{2} \theta\right],
$$

in terms of the total modular weights

$$
\begin{aligned}
& n_{\alpha}=3\left(\sum_{r} n_{\alpha r} \Theta_{r}^{2}+\sum_{s} n_{\alpha s} \Theta_{s}^{2}\right), \\
& n_{x}=3\left(\sum_{r} n_{x r} \Theta_{r}^{2}+\sum_{s} n_{x s} \Theta_{s}^{2}\right) .
\end{aligned}
$$

In the particular case where a single anomalous $U(1)$ is present, without any extra nonanomalous $U(1)$, eq. (6.10) simplifies to ${ }^{4}$

$$
\frac{m_{\alpha}^{2}}{m_{3 / 2}^{2}}=1+\left(n_{\alpha}-\frac{q_{\alpha}}{q_{x}} n_{x}\right) \cos ^{2} \theta-3 \frac{q_{\alpha}}{q_{x}} \sin ^{2} \theta .
$$

The formula (6.13) shows that the soft scalar masses are universal for an arbitrary $\theta$ only if both the modular weights $n_{\alpha}$ and the $U(1)$ charges $q_{\alpha}$ are universal. In the dilaton domination limit $\theta \rightarrow \pi / 2$, it is enough that the charges $q_{\alpha}$ are universal. This situation

\footnotetext{
${ }^{4}$ The expression (6.13) differs from the one derived in $[22,23,24]$ by the coefficient and the dependence on the Goldstino angle of the last term, which plays an important role for the issue of flavor universality. This can be traced back to the fact that the expression obtained by applying eq. (4.16) for the values of the auxiliary components of the Higgs fields differs from the one used in [22, 23, 23], where the dependence on $H^{x}$ implied by gauge invariance in the superpotential stabilizing $S$ was not taken into account.
} 
is however excluded by metastability considerations. On the other hand, in the geometric moduli domination limit $\theta \rightarrow 0$, it is enough that the combination $n_{\alpha}-q_{\alpha} / q_{x} n_{x}$, acting as an effective modular weight, is universal. This situation is allowed by metastability consideration, and we will consider it as our preferred scenario. Summarizing, one could conceivably achieve universal soft masses if $\theta \rightarrow 0$ and the differences between the modular weights and the charges of any pair of different flavors of matter fields satisfy:

$$
\Delta n-\frac{\Delta q}{q_{x}} n_{x}=0 .
$$

It is now worth to comment on what happens in the situation of [20,21], where the $U(1)$ symmetries play the role of flavor symmetries. In these works it was shown that a nonzero perturbative Yukawa coupling mixing two flavors can arise from a gauge-invariant and modular-invariant higher-dimensional operator involving powers of the gauge symmetry breaking fields $H^{x}$, provided that the differences between the modular weights and the charges of the two involved matter fields satisfy a selection rule which is precisely given by eq. (6.14). In such a situation, the contribution to the soft scalar masses that we have computed above would display an improved structure, with the difference between two different flavors given simply by $\Delta m^{2} / m_{3 / 2}^{2}=-3 \Delta n / n_{x} \sin ^{2} \theta^{5}$. In the volume domination limit $\theta \rightarrow 0$, one finds in particular universal diagonal values for the soft scalar mass matrix. However, it must be emphasized that the relation (6.14) also allows the appearance of gauge-invariant and modular-invariant higher-dimensional operators in the Kähler potential mixing two different families of matter fields and powers of the gauge symmetry breaking fields $H^{x}$. These would induce subleading off-diagonal elements for the soft mass matrix, which are as dangerous as differences between diagonal elements, and represent the main difficulty in constructing viable supersymmetric flavor models. It would nevertheless be interesting to further explore whether this somewhat peculiar situation can help in getting a simple and satisfactory supersymmetric flavor model in this framework, with the anomalous $U(1)$ symmetry, possibly together with other nonanomalous but Higgsed $U(1)$ symmetries, playing the role of flavor symmetries.

\subsection{Brane models}

Let us consider now the case of intersecting brane models $[47,48,49]$. In these models, there can be several anomalous $U(1)$ 's, and the Green-Schwarz mechanism compensating them involves linear combinations of $S$ and the $U_{s}$, but not the $T_{r}$. In this case, the Higgs fields compensating the Fayet-Iliopoulos terms induced by the anomalous $U(1)$ 's can be either additional matter fields $H^{x}$ with a canonical Kähler potential and a moduli-

\footnotetext{
${ }^{5}$ This result differs qualitatively from the one derived [20,21], as far as the dependence on the Goldstino angle is concerned. We believe that this is due to the fact that the simple minimization procedure used in $[20,21]$ to derive the value of the vector auxiliary field also implicitly neglects the fact that gauge invariance implies a non-trivial dependence of the $F$-term potential on the Higgs fields, which does not amount just to their soft mass terms.
} 
dependent wave-function factor, or the complex structure moduli $U_{s}$, which have a noncanonical Kähler potential ${ }^{6}$.

In the case where all the moduli $S, T_{r}$ and $U_{s}$ are stabilized by $F$-term effects and the $D$-terms are compensated by canonical matter Higgs fields $H^{x}$, the situation is of the type discussed in section 4, and the general form of the Kähler potential is given by eq. (4.1). The potential of the moduli has the simple form:

$$
\hat{K}=-\ln \left(S+\bar{S}-\sum_{a} \delta_{a} V^{a}\right)-\sum_{r} \ln \left(T_{r}+\bar{T}_{r}\right)-\sum_{s} \ln \left(U_{s}+\bar{U}_{s}-\sum_{a} \delta_{s a} V^{a}\right)
$$

The form of the relevant couplings between the moduli fields and the matter and Higgs fields have been studied only very recently in the literature $[52,53]$. Their precise form is still under debate [54], and does not seem to rest on any symmetry argument. For this reason, we shall leave it arbitrary and take:

$$
\begin{aligned}
& K_{\alpha \bar{\alpha}}=K_{\alpha \bar{\alpha}}\left(T_{r}+\bar{T}_{r}, U_{s}+\bar{U}_{s}-\sum_{a} \delta_{s a} V^{a}\right), \\
& \tilde{K}_{x \bar{x}}=\tilde{K}_{x \bar{x}}\left(T_{r}+\bar{T}_{r}, U_{s}+\bar{U}_{s}-\sum_{a} \delta_{s a} V^{a}\right) .
\end{aligned}
$$

We can however still parametrize the corresponding mixed components of the Riemann tensor as $R_{\alpha \bar{\alpha} I \bar{J}}=-n_{\alpha i \bar{\jmath}}$ and $R_{X \bar{X} I \bar{J}}=-n_{x i \bar{\jmath}}$. The quantities $n_{\alpha i \bar{\jmath}}$ and $n_{x i \bar{\jmath}}$ play then the role of effective modular weights. In general they can depend on the moduli fields and have non-vanishing off-diagonal components. The structure of the $F$-term contribution to soft terms is consequently more complicated in this case $[55,56]$.

We can now apply the results obtained in section 4 to derive the physically normalized soft masses. The result can be written in terms of the above-defined effective modular weights and reads

$$
\frac{m_{\alpha}^{2}}{m_{3 / 2}^{2}}=\left[1+n_{\alpha} \cos ^{2} \theta\right]-\sum_{a, x} q_{\alpha a} q_{a x}^{-1}\left[\left(n_{x}+b_{x}\right) \cos ^{2} \theta+a_{x} \sin ^{2} \theta+c_{x} \sin 2 \theta\right],
$$

in terms of the total modular weights

$$
\begin{aligned}
& n_{\alpha}=3\left(\sum_{r, r^{\prime}} n_{\alpha r r^{\prime}} \Theta_{r} \Theta_{r^{\prime}}+\sum_{s, s^{\prime}} n_{\alpha s s^{\prime}} \Theta_{s} \Theta_{s^{\prime}}\right), \\
& n_{x}=3\left(\sum_{r, r^{\prime}} n_{x r r^{\prime}} \Theta_{r} \Theta_{r^{\prime}}+\sum_{s, s^{\prime}} n_{x s s^{\prime}} \Theta_{s} \Theta_{s^{\prime}}\right) .
\end{aligned}
$$

\footnotetext{
${ }^{6}$ It is useful to recall that the angles defining the geometric orientation of the branes are determined by the VEV and the shift charges of $S$ and $U_{s}$, whereas the nature of the intersection is possibly influenced by localized Higgs fields $H^{x}[48,49,50,51]$. The condition for approximate supersymmetry that the sources induced for the $D$-terms by the fields $S$ and $U_{s}$ vanish translates then into the conditions that the relative angles between the branes should correspond to rotations belonging to an $S U(3)$ subgroup of $S O(6)$ and preserving a $U(1)$ factor. The two situations described above can then be interpreted as follows. In the first case, the values of the angles are fixed by some $F$-term dynamics and violate supersymmetry; the VEV of the additional Higgs fields can then be geometrically interpreted as corresponding to a recombination of the branes through their intersections to a final state with new angles preserving approximately supersymmetry. In the second case, the values of the angles are instead fixed by the $D$-term dynamics itself, and do thus approximately preserve supersymmetry from the beginning.
} 
and the following functions of the variables (4.37) depending on the inverse VEV of the moduli fields:

$$
\begin{aligned}
& a_{x}=6 \frac{t_{S x}}{t_{S x}+\sum_{s} t_{U_{s} x}}-3\left(\frac{t_{S x}}{t_{S x}+\sum_{s} t_{U_{s} x}}\right)^{2}, \\
& b_{x}=6 \sum_{s} \frac{t_{U_{s} x}}{t_{S x}+\sum_{s} t_{U_{s} x}} \Theta_{s}^{2}-3\left(\sum_{s} \frac{t_{U_{s} x}}{t_{S x}+\sum_{s} t_{U_{s} x}} \Theta_{s}\right)^{2}, \\
& c_{x}=-3\left(\frac{t_{S x}}{t_{S x}+\sum_{s} t_{U_{s} x}}\right)\left(\sum_{s} \frac{t_{U_{s} x}}{t_{S x}+\sum_{s} t_{U_{s} x}} \Theta_{s} \cos \left(\gamma_{s}-\gamma\right)\right) .
\end{aligned}
$$

In the particular case where a single anomalous $U(1)$ is present, without any additional anomalous or non-anomalous $U(1)$ and only one Higgs field, the above expression simplifies and yields

$$
\frac{m_{\alpha}^{2}}{m_{3 / 2}^{2}}=1+\left(n_{\alpha}-\frac{q_{\alpha}}{q_{x}}\left(n_{x}+b_{x}\right)\right) \cos ^{2} \theta-\frac{q_{\alpha}}{q_{x}}\left(a_{x} \sin ^{2} \theta+c_{x} \sin 2 \theta\right) .
$$

The formula (6.22) shows that the soft scalar masses are as before universal for an arbitrary $\theta$ only if both the modular weights $n_{\alpha}$ and the $U(1)$ charges $q_{\alpha}$ are universal. In the dilaton domination limit $\theta \rightarrow \pi / 2$, it is enough that the charges $q_{\alpha}$ are universal, but this situation is again excluded by metastability considerations. In the geometric moduli domination limit $\theta \rightarrow 0$, on the other hand, it is enough that the corrected modular weight $n_{\alpha}-q_{\alpha} / q_{x}\left(n_{x}+b_{x}\right)$ is universal. Since $n_{\alpha}$ and $n_{x}$ may have related functional forms, but $b_{x}$ is instead an unrelated function, this universality could plausibly arise only in situations where $b_{x}=0$. This happens for instance in the case where all the complex structure moduli are stabilized in a supersymmetry way, so that $\Theta_{s}=0$. Summarizing, one could conceivably achieve universal soft masses if $\theta \rightarrow 0, \Theta_{s} \rightarrow 0$, and the differences between the effective modular weights and the charges of any pair of different flavors of matter fields satisfy:

$$
\Delta n-\frac{\Delta q}{q_{x}} n_{x}=0
$$

In this situation, supersymmetry would be dominated only by the Kähler moduli. Since there are 3 of them, the metastability condition is then marginally violated, and subleading corrections to the Kähler potential are expected to play a crucial role in the stabilization of the lightest modulus [10].

In the case where only the $S$ and $T_{r}$ moduli are stabilized by $F$-term effects, whereas the $U_{s}$ moduli are stabilized by $D$-terms and act as Higgs fields, the situation is that of section 5 , and the general form of the Kähler potential is given by eq. (5.1). The potential of the supersymmetry breaking moduli and the Higgs fields have the forms:

$$
\begin{aligned}
\hat{K} & =-\ln \left(S+\bar{S}-\sum_{a} \delta_{a} V^{a}\right)-\sum_{r} \ln \left(T_{r}+\bar{T}_{r}\right), \\
\tilde{K} & =-\sum_{s} \ln \left(U_{s}+\bar{U}_{s}-\sum_{a} \eta_{s a} V^{a}\right) .
\end{aligned}
$$


The coupling between the moduli fields and the matter fields is again left arbitrary, whereas the coupling between the moduli fields and the Higgs fields is in this case absent:

$$
K_{\alpha \bar{\alpha}}=K_{\alpha \bar{\alpha}}\left(T_{r}+\bar{T}_{r}, U_{s}+\bar{U}_{s}-\sum_{a} \eta_{s a} V^{a}\right) .
$$

As before, we can still parametrize the mixed components of the Riemann tensor as $R_{\alpha \bar{\alpha} I \bar{J}}=-n_{\alpha i \bar{\jmath}}$, where $n_{\alpha i \bar{\jmath}}$ is some effective modular weight depending on the moduli fields and possessing non-vanishing off-diagonal entries. On the other hand, $R_{X \bar{X} I \bar{J}}=0$ in this case.

We can now apply the results obtained in section 5 to derive the physically normalized soft masses. As discussed in section 5, the $D$-term contribution to the soft masses vanishes in this case. One finds then simply:

$$
\frac{m_{\alpha}^{2}}{m_{3 / 2}^{2}}=\left[1+n_{\alpha} \cos ^{2} \theta\right]
$$

in terms of the total modular weights

$$
n_{\alpha}=3\left(\sum_{r, r^{\prime}} n_{\alpha r r^{\prime}} \Theta_{r} \Theta_{r^{\prime}}+\sum_{s, s^{\prime}} n_{\alpha s s^{\prime}} \Theta_{s} \Theta_{s^{\prime}}\right) .
$$

This result is in particular valid in the minimal situation involving three anomalous $U(1)$, whose $D$-terms stabilize the three complex structure moduli $U_{s}$ relative to the dilaton $S$.

As mentioned at the end of section 5, there is in this case a restriction on the Goldstino direction. Indeed, the bosonic components of the 3 complex structure multiplets $U_{s}$ are completely determined in terms of the those of the dilaton $S$. More precisely, from the $3 D$-flatness conditions one finds the relations $\left(U_{s}+\bar{U}_{s}\right)^{-1}=\left(\sum_{a} Z_{s a}^{-1}\right)(S+\bar{S})^{-1}$ and $F_{U_{s}}=\left(\sum_{a} Z_{s a}^{-1}\right) F_{S}$, in terms of the 3 by 3 matrix $Z_{s a}=-\eta_{s a} / \delta_{a}$. This implies then that the rescaled auxiliary fields satisfy the simple relation $F^{\bar{U}_{s}}=F^{\bar{S}}$. Comparing with the general parametrization of eqs. (6.2) and (6.3), this implies the restriction

$$
\Theta_{s}=\tan \theta
$$

The formula (6.27) shows that the soft scalar masses are in this case insensitive to the $U(1)$ charges $q_{\alpha a}$, and are universal for an arbitrary $\theta$ if the modular weights $n_{\alpha}$ are universal. Notice finally that in the geometric moduli domination limit $\theta \rightarrow 0$ nothing really special happens for the soft masses, but the relation (6.29) implies then that the complex structure moduli give a negligible effect as well and only the Kähler moduli break supersymmetry. Since there are 3 of these, the metastability condition is then again marginally violated, implying that subleading corrections to the Kähler potential are expected to play a crucial role in stabilizing at least one of them [10].

\section{Conclusions}

In this paper, we have studied in some generality the structure of soft scalar masses in superstring models with heavy anomalous $U(1)$ vector fields. We have considered the 
minimal situation where the sources induced by the supersymmetry breaking fields in the $D$-terms are approximately compensated, at leading order in $m_{3 / 2}$, by some Higgs fields taking suitable VEV. We have computed with two different methods the structure of the $D$-term contribution to soft scalar masses, relative to the usual $F$-term effect. We have shown that the result significantly depends on the functional form of the Kähler potential for the Higgs fields. In particular, assuming that the supersymmetry breaking fields are untwisted moduli fields with a logarithmic Kähler potential, we find that the effect of heavy vector fields is non-trivial when the Higgs fields are matter fields with a quadratic Kähler potential, but can accidentally vanish if they happen to be moduli fields with a logarithmic Kähler potential.

For heterotic orbifold models, we find a result for the soft scalar masses that slightly differs from previous analyses. We assume that the supersymmetry breaking moduli $S$, $T_{r}$ and $U_{s}$ are stabilized by some $F$-term effects and that the $D$-terms are approximately compensated by a minimal set of matter Higgs fields $H^{x}$ with a quadratic Kähler potential. The resulting soft scalar masses have then a pretty simple structure, with a non-trivial $D$-term contribution that is as important as the $F$-term contribution. The total masses depend on the Goldstino direction of supersymmetry breaking, but not on the scalar VEV of the supersymmetry breaking fields. They can be flavor-universal independently of any further assumption about the superpotential and the Goldstino direction only under the very strong condition that both the modular weights $n_{\alpha}$ and the $U(1)$ charges $q_{\alpha a}$ of the matter fields are universal. On the other hand, in the geometric moduli domination limit, which on the contrary of the dilaton mediation limit is compatible with flatness and metastability of the vacuum, flavor universality of the soft masses is guaranteed under the milder requirement that suitable linear combinations of modular weights and $U(1)$ charges are universal.

For intersecting brane models, on the other hand, we find new results displaying a richer variety of possibilities. In this case, there exist two qualitatively different options for the stabilization of the various fields, leading to radically different structures for the soft scalar masses. A first possibility is that all the supersymmetry breaking moduli $S, T_{r}$ and $U_{s}$ are stabilized by $F$-term effects and that the $D$-terms are approximately compensated by a minimal set of matter Higgs fields $H^{x}$ with a quadratic Kähler potential, much as in heterotic models. The resulting soft scalar masses have then again a non-trivial $D$-term contribution that is comparable to the $F$-term contribution. The total masses depend in this case not only on the Goldstino direction of supersymmetry breaking, but also on the scalar VEV of the supersymmetry breaking fields. As for heterotic models, they can be flavor universal without any tuning only if both the modular weights $n_{\alpha}$ and the $U(1)$ charges $q_{\alpha a}$ are universal. On the other hand, in the geometric moduli domination limit, it is enough that certain linear combinations of the latter are universal. A second possibility is that only the $S$ and $T_{r}$ supersymmetry breaking moduli are stabilized by $F$-term effects, whereas the $U_{s}$ moduli behave as a minimal set of Higgs fields with a logarithmic Kähler potential and are stabilized by the $D$-terms in such a way to 
approximately compensate them. In this radically different situation, the resulting soft scalar masses have an accidentally vanishing $D$-term contribution and are thus entirely controlled by the $F$-term contribution. The total masses depend also in this case on both the Goldstino direction of supersymmetry breaking and the scalar VEV of the supersymmetry breaking fields. The interesting novelty arising in this situation is that these masses can be flavor universal without any tuning under the mild constraint that these effective modular weights $n_{\alpha}$ are universal, independently of the $U(1)$ charges $q_{\alpha a}$. On the other hand, the Goldstino direction turns out to be constrained by gauge invariance. In the geometric moduli domination limit, no further simplification occurs in the soft masses, but the $T_{r}$ moduli dominate over the $U_{s}$ moduli.

We should finally emphasized that we have restricted our attention to situations involving a minimal set of Higgs fields, which do at the same time the jobs of compensating the $D$-term potential and making the superpotential of the moduli gauge invariant. In this case, the effects of the Higgs fields can be entirely related to those of the moduli fields; as a consequence, the soft masses can then be parametrized in terms of the arbitrary $F$ terms of the sole moduli multiplets. One may however consider also more general non-minimal situations, where two different sets of Higgs fields are used respectively to compensate the $D$-term potential and to make the superpotential of the moduli gauge invariant. In that case, the effects of the Higgs fields will not be entirely determined by those of the moduli fields, and the extra Higgs multiplets will behave as additional fields of the hidden sector, together with the moduli fields; the soft masses will then depend on the arbitrary $F$ terms of not only the moduli but also the extra Higgs multiplets. In other words, whenever more charged fields than gauge symmetries are involved in the hidden sector dynamics, the number of light gauge-invariant chiral multiplets participating to supersymmetry breaking in the low-energy theory increases. One may then consider the particular limit in which these extra degrees of freedom decouple at the scale of supersymmetry breaking, for example because of a large mass preserving supersymmetry. In that case, one should then recover the same situation as for a minimal set of Higgs fields. This means that the minimal situation considered in this paper can actually be viewed as the most general situation compatible with the assumption that the hidden sector involves only the moduli.

\section{Acknowledgments}

We thank R. Rattazzi and A. Uranga for many enlightening discussions. We are also grateful to B. de Carlos, E. Dudas, M. Gomez-Reino and F. Zwirner for useful comments. This work was supported by the Swiss National Science Foundation. 


\section{References}

[1] R. Barbieri, S. Ferrara and C. A. Savoy, Gauge models with spontaneously broken local supersymmetry, Phys. Lett. B 119 (1982) 343.

[2] H. P. Nilles, M. Srednicki and D. Wyler, Weak interaction breakdown induced by supergravity, Phys. Lett. B 120 (1983) 346.

[3] L. J. Hall, J. D. Lykken and S. Weinberg, Supergravity as the messenger of supersymmetry breaking, Phys. Rev. D 27 (1983) 2359.

[4] A. H. Chamseddine, R. Arnowitt and P. Nath, Locally supersymmetric grand unification, Phys. Rev. Lett. 49 (1982) 970.

[5] L. E. Ibanez, Locally supersymmetric SU(5) grand unification, Phys. Lett. B 118 (1982) 73.

[6] N. Ohta, Grand unified theories based on local supersymmetry, Prog. Th. Phys. 70 (1983) 542.

[7] V. S. Kaplunovsky and J. Louis, Model independent analysis of soft terms in effective supergravity and in string theory, Phys. Lett. B 306 (1993) 269 [hep-th/9303040].

[8] A. Brignole, L. E. Ibanez and C. Munoz, Towards a theory of soft terms for the supersymmetric Standard Model, Nucl. Phys. B 422 (1994) 125 [Errat. B 436 (1995) 747] [hep-ph/9308271].

[9] A. Brignole, L. E. Ibanez, C. Munoz and C. Scheich, Some issues in soft SUSY breaking terms from dilaton / moduli sectors, Z. Phys. C 74 (1997) 157 [hep-ph/9508258].

[10] M. Gomez-Reino and C. A. Scrucca, Locally stable non-supersymmetric Minkowski vacua in supergravity, JHEP 0605 (2006) 015 [hep-th/0602246].

[11] M. Gomez-Reino and C. A. Scrucca, Constraints for the existence of flat and stable nonsupersymmetric vacua in supergravity, JHEP 0609 (2006) 008 [hep-th/0606273].

[12] M. Gomez-Reino and C. A. Scrucca, Metastable supergravity vacua with F and D supersymmetry breaking JHEP 0708 (2007) 091 [arXiv:0706.2785].

[13] M. B. Green and J. H. Schwarz, Anomaly cancellation in supersymmetric D=10 gauge theory and superstring theory, Phys. Lett. B 149 (1984) 117.

[14] M. Dine, N. Seiberg and E. Witten, Fayet-Iliopoulos terms in string theory, Nucl. Phys. B 289 (1987) 589.

[15] M. Drees, Intermediate Scale Symmetry Breaking and the Spectrum of Super Partners in Superstring Inspired Supergravity Models, Phys. Lett. B 181 (1986) 279.

[16] J. S. Hagelin and S. Kelley, Sparticle masses as a probe of GUT physics, Nucl. Phys. B 342 (1990) 95.

[17] P. Binetruy and E. Dudas, Gaugino condensation and the anomalous U(1), Phys. Lett. B 389 (1996) 503 [hep-th/9607172].

[18] G. R. Dvali and A. Pomarol, Anomalous U(1) as a mediator of supersymmetry breaking, Phys. Rev. Lett. 77 (1996) 3728 [hep-ph/9607383].

[19] T. Barreiro, B. de Carlos, J. A. Casas and J. M. Moreno, Anomalous U(1), gaugino condensation and supergravity, Phys. Lett. B 445 (1998) 82 [hep-ph/9808244]. 
[20] E. Dudas, S. Pokorski and C. A. Savoy, Soft scalar masses in supergravity with horizontal $U(1)_{X}$ gauge symmetry, Phys. Lett. B 369 (1996) 255 [hep-ph/9509410].

[21] E. Dudas, C. Grojean, S. Pokorski and C. A. Savoy, Abelian flavour symmetries in supersymmetric models, Nucl. Phys. B 481 (1996) 85 [hep-ph/9606383].

[22] Y. Kawamura and T. Kobayashi, Soft scalar masses in string models with anomalous U(1) symmetry, Phys. Lett. B 375 (1996) 141, [Errat. B 388 (1996) 867] [hep-ph/9601365].

[23] Y. Kawamura and T. Kobayashi, Generic formula of soft scalar masses in string models, Phys. Rev. D 56 (1997) 3844 [hep-ph/9608233].

[24] T. Higaki, Y. Kawamura, T. Kobayashi and H. Nakano, Anomalous U(1) D-term contribution in type I string models, Phys. Rev. D 69 (2004) 086004 [hep-ph/0308110].

[25] N. Arkani-Hamed, M. Dine and S. P. Martin, Dynamical supersymmetry breaking in models with a Green-Schwarz mechanism, Phys. Lett. B 431 (1998) 329 [hep-ph/9803432].

[26] K. Choi and K. S. Jeong, Supersymmetry breaking and moduli stabilization with anomalous U(1) gauge symmetry, JHEP 0608 (2006) 007 [hep-th/0605108].

[27] E. Cremmer, B. Julia, J. Scherk, S. Ferrara, L. Girardello and P. van Nieuwenhuizen, Spontaneous symmetry breaking and higgs effect in supergravity without cosmological constant, Nucl. Phys. B 147 (1979) 105.

[28] E. Witten and J. Bagger, Quantization of Newton's constant in certain supergravity theories, Phys. Lett. B 115 (1982) 202.

[29] E. Cremmer, S. Ferrara, L. Girardello and A. Van Proeyen, Coupling supersymmetric YangMills theories to supergravity, Phys. Lett. B 116 (1982) 231.

[30] E. Cremmer, S. Ferrara, L. Girardello and A. Van Proeyen, Yang-Mills theories with local supersymmetry: Lagrangian, transformation laws and superhiggs effect, Nucl. Phys. B 212 (1983) 413.

[31] J. A. Bagger, Coupling the gauge invariant supersymmetric nonlinear sigma model to supergravity, Nucl. Phys. B 211 (1983) 302.

[32] S. Ferrara, C. Kounnas and F. Zwirner, Mass formulae and natural hierarchy in string effective supergravities, Nucl. Phys. B 429 (1994) 589 [Errat. B 433 (1995) 255] [hep-th/9405188].

[33] E. Dudas and S. K. Vempati, Large D-terms, hierarchical soft spectra and moduli stabilisation, Nucl. Phys. B 727 (2005) 139 [hep-th/0506172].

[34] Y. Kawamura, Model independent analysis of soft masses in heterotic string models with anomalous U(1) symmetry, Phys. Lett. B 446 (1999) 228 [hep-ph/9811312].

[35] J. R. Ellis, C. Kounnas and D. V. Nanopoulos, No Scale Supersymmetric Guts, Nucl. Phys. B 247 (1984) 373.

[36] L. Randall and R. Sundrum, Out of this world supersymmetry breaking Nucl. Phys. B 557 (1999) 79 [hep-th/9810155].

[37] A. Pomarol and S. Dimopoulos, Superfield derivation of the low-energy effective theory of softly broken supersymmetry, Nucl. Phys. B 453 (1995) 83 [hep-ph/9505302]. 
[38] R. Rattazzi, A Note on the effective soft SUSY breaking Lagrangian below the GUT scale, Phys. Lett. B 375 (1996) 181 [hep-ph/9507315].

[39] E. Witten, Dimensional Reduction Of Superstring Models, Phys. Lett. B 155 (1985) 151.

[40] S. Ferrara, C. Kounnas and M. Porrati, General dimensional reduction of ten-dimensional supergravity and superstring, Phys. Lett. B 181 (1986) 263.

[41] M. Cvetic, J. Louis and B. A. Ovrut, A string calculation of the Kahler potentials for moduli of Z(N) orbifolds, Phys. Lett. B 206 (1988) 227.

[42] R. Brustein and S. P. de Alwis, Moduli potentials in string compactifications with fluxes: mapping the discretuum, Phys. Rev. D 69 (2004) 126006 [hep-th/0402088].

[43] L. J. Dixon, J. A. Harvey, C. Vafa and E. Witten, Strings on orbifolds, Nucl. Phys. B 261 (1985) 678.

[44] L. J. Dixon, J. A. Harvey, C. Vafa and E. Witten, Strings on orbifolds. 2, Nucl. Phys. B 274 (1986) 285.

[45] L. J. Dixon, V. Kaplunovsky and J. Louis, On effective field theories describing (2,2) vacua of the heterotic string, Nucl. Phys. B 329 (1990) 27.

[46] L. E. Ibanez and D. Lust, Duality anomaly cancellation, minimal string unification and the effective low-energy Lagrangian of 4-D strings, Nucl. Phys. B 382 (1992) 305 [hep-th/9202046].

[47] G. Aldazabal, S. Franco, L. E. Ibanez, R. Rabadan and A. M. Uranga, Intersecting brane worlds, JHEP 0102 (2001) 047 [hep-ph/0011132].

[48] M. Cvetic, G. Shiu and A. M. Uranga, Three-family supersymmetric standard like models from intersecting brane worlds, Phys. Rev. Lett. 87 (2001) 201801 [hep-th/0107143].

[49] M. Cvetic, G. Shiu and A. M. Uranga, Chiral four-dimensional $N=1$ supersymmetric type IIA orientifolds from intersecting D6-branes, Nucl. Phys. B 615 (2001) 3 [hep-th/0107166].

[50] D. Cremades, L. E. Ibanez and F. Marchesano, SUSY quivers, intersecting branes and the modest hierarchy problem, JHEP 0207 (2002) 009 [hep-th/0201205].

[51] G. Villadoro and F. Zwirner, D terms from D-branes, gauge invariance and moduli stabilization in flux compactifications, JHEP 0603 (2006) 087 [hep-th/0602120].

[52] D. Lust, P. Mayr, R. Richter and S. Stieberger, Scattering of gauge, matter, and moduli fields from intersecting branes, Nucl. Phys. B 696 (2004) 205 hep-th/0404134].

[53] M. Bertolini, M. Billo, A. Lerda, J. F. Morales and R. Russo, Brane world effective actions for D-branes with fluxes, Nucl. Phys. B 743 (2006) 1 [hep-th/0512067].

[54] R. Blumenhagen, B. Kors, D. Lust and S. Stieberger, Four-dimensional string compactifications with D-Branes, orientifolds and fluxes, Phys. Rept. 445 (2007) 1 [hep-th/0610327].

[55] D. Lust, S. Reffert and S. Stieberger, MSSM with soft SUSY breaking terms from D7-branes with fluxes, Nucl. Phys. B 727 (2005) 264 [hep-th/0410074].

[56] A. Font and L. E. Ibanez, SUSY-breaking soft terms in a MSSM magnetized D7-brane model, JHEP 0503 (2005) 040 [hep-th/0412150]. 